\title{
Numerical renormalization group calculation of impurity internal energy and specific heat of quantum impurity models
}

\author{
L. Merker and T. A. Costi \\ Peter Grünberg Institut and Institute for Advanced Simulation, Research Centre Jülich, 52425 Jülich, Germany
}

(Received 22 June 2012; published 28 August 2012)

\begin{abstract}
We introduce a method to obtain the specific heat of quantum impurity models via a direct calculation of the impurity internal energy requiring only the evaluation of local quantities within a single numerical renormalization group (NRG) calculation for the total system. For the Anderson impurity model we show that the impurity internal energy can be expressed as a sum of purely local static correlation functions and a term that involves also the impurity Green function. The temperature dependence of the latter can be neglected in many cases, thereby allowing the impurity specific heat $C_{\mathrm{imp}}$ to be calculated accurately from local static correlation functions; specifically via $C_{\mathrm{imp}}=\frac{\partial E_{\text {ionic }}}{\partial T}+\frac{1}{2} \frac{\partial E_{\mathrm{hyb}}}{\partial T}$, where $E_{\text {ionic }}$ and $E_{\mathrm{hyb}}$ are the energies of the (embedded) impurity and the hybridization energy, respectively. The term involving the Green function can also be evaluated in cases where its temperature dependence is non-negligible, adding an extra term to $C_{\text {imp }}$. For the nondegenerate Anderson impurity model, we show by comparison with exact Bethe ansatz calculations that the results recover accurately both the Kondo induced peak in the specific heat at low temperatures as well as the high-temperature peak due to the resonant level. The approach applies to multiorbital and multichannel Anderson impurity models with arbitrary local Coulomb interactions. An application to the Ohmic two-state system and the anisotropic Kondo model is also given, with comparisons to Bethe ansatz calculations. The approach could also be of interest within other impurity solvers, for example, within quantum Monte Carlo techniques.
\end{abstract}

DOI: 10.1103/PhysRevB.86.075150

PACS number(s): 75.20.Hr, 71.27.+a, 72.15.Qm

\section{INTRODUCTION}

Quantum impurity models play an important role in condensed matter physics, for example, as models of transition metal and rare-earth impurities in metals ${ }^{1}$ or two-level systems $^{2-6}$ and qubits ${ }^{7}$ interacting with an environment or in describing the Kondo effect in nanoscale devices such as molecular transistors, ${ }^{8-11}$ semiconductor quantum dots, ${ }^{12-14}$ carbon nanotubes, ${ }^{15}$ and magnetic ions such as $\mathrm{Co}^{16,17}$ or $\mathrm{Ce}^{18}$ adsorbed on surfaces. In addition, they appear as the effective models within dynamical mean field theory (DMFT) treatments of strongly correlated electron systems, such as heavy fermions and transition metal oxides. ${ }^{19-22}$ Hence, new approaches to calculate their dynamic, thermodynamic, and transport properties are potentially of wide interest.

The numerical renormalization group (NRG) method, ${ }^{23-26}$ in particular, has proven very successful for the study of quantum impurity models. The method, described briefly in the next section, gives both the thermodynamic, ${ }^{23-25,27}$ dynamic, ${ }^{28-35}$ and transport properties ${ }^{36}$ of quantum impurities. Thermodynamic properties, such as the specific heat, are of particular interest for bulk systems, such as dilute concentrations of transition metal or rare-earth ions in nonmagnetic metals. ${ }^{1}$ A measurement of the temperature dependence of the specific heat or susceptibility of such systems provides important information about their physical behavior, for example, whether such systems exhibit Fermi liquid or non-Fermi liquid behavior at low temperature and thus information about the nature of their low-energy excitations. ${ }^{37,38}$

The usual approach to calculating the specific heat of quantum impurity models within the NRG method consists of a two-stage procedure ${ }^{24-27}$ in which the Hamiltonians of the total system $H$ is first diagonalized, followed by a similar diagonalization for the host Hamiltonian $H_{0}$. Here, $H=$ $H_{\text {imp }}+H_{\text {int }}+H_{0}$ is the Hamiltonian of a quantum impurity (described by $H_{\text {imp }}$ ), interacting with a host (described by $H_{0}$ ) via the interaction term $H_{\text {int }}$. From the eigenvalues of $H$ and $H_{0}$, the grand canonical partition functions $Z=\operatorname{Tr} e^{-\beta H}$ and $Z_{0}=\operatorname{Tr} e^{-\beta H_{0}}$ and the corresponding thermodynamic potentials $\Omega(T)=-k_{\mathrm{B}} T \ln Z$ and $\Omega_{0}(T)=-k_{\mathrm{B}} T \ln Z_{0}$ are constructed, where $\beta=1 / k_{\mathrm{B}} T$ is the inverse temperature. The impurity contribution to the specific heat $C_{\mathrm{imp}}(T)$ is then obtained by subtraction via $C_{\text {imp }}(T)=C(T)-C_{0}(T)$, where $C(T)$ and $C_{0}(T)$ are the specific heats of the total system and of the host system, respectively,

$$
\begin{aligned}
C(T) & =-T \frac{\partial^{2} \Omega(T)}{\partial T^{2}}=k_{\mathrm{B}} \beta^{2}\left\langle(H-\langle H\rangle)^{2}\right\rangle, \\
C_{0}(T) & =-T \frac{\partial^{2} \Omega_{0}(T)}{\partial T^{2}}=k_{\mathrm{B}} \beta^{2}\left\langle\left(H_{0}-\left\langle H_{0}\right\rangle\right)^{2}\right\rangle, \\
C_{\mathrm{imp}}(T) & =C(T)-C_{0}(T) .
\end{aligned}
$$

In this paper we present a new approach to the calculation of the impurity internal energy and specific heat of quantum impurity models within the numerical renormalization group (NRG) method. ${ }^{23-26}$ It relies on expressing the impurity internal energy in terms of local quantities, and as such is not restricted to the NRG but may be implemented within any impurity solver that calculates such quantities. The main result of this paper is the (approximate) expression for the impurity specific heat of the Anderson model (see Sec. III)

$$
C_{\text {imp }}(T)=\frac{\partial E_{\text {ionic }}}{\partial T}+\frac{1}{2} \frac{\partial E_{\text {hyb }}}{\partial T},
$$

where $E_{\text {ionic }}=\left\langle H_{\text {imp }}\right\rangle$ and $E_{\text {hyb }}=\left\langle H_{\text {int }}\right\rangle$. The main advantages of this approach are that (i) Eq. (4) involves only a first temperature derivative and is expected to be more accurate for numerical evaluations than Eqs. (1)-(3) which involve a second temperature derivative of the thermodynamic potential, 
or the calculation of the total energy fluctuation; (ii) the host contribution to the internal energy $\left\langle H_{0}\right\rangle$ has been analytically subtracted out (see Sec. III), so only the diagonalization of $H$ is required; (iii) only local static correlation functions appearing in $\left\langle H_{\text {imp }}\right\rangle$ and $\left\langle H_{\text {int }}\right\rangle$ are required; and (iv) as we shall show, the new approach is less sensitive to discretization effects of the host than the usual approach which evaluates expectation values of extensive quantities. We illustrate the method by applying it to the Anderson impurity model and we compare the results for specific heats with those from the conventional NRG approach ${ }^{27,36,39}$ and with exact results from thermodynamic Bethe ansatz calculations. ${ }^{40-42}$

Early approaches to the specific heat of dilute Kondo systems used an equation of motion decoupling scheme for the Kondo model ${ }^{43}$ and expressed the impurity internal energy in terms of the local T matrix. The results obtained for the specific heat within this approximation were inadequate, violating, for example, Fermi liquid properties at low temperatures. ${ }^{44}$ A formally exact expression for the internal energy of the Anderson model, in terms of the local self-energy and the local Green function, was obtained by Kjöllerström et al. in Ref. 45. They evaluated the specific heat in the low-density limit (corresponding to a small occupation of the local level) obtaining correct results obeying Fermi liquid theory in this limit.

The most reliable approaches to specific heats of quantum impurity models are the Bethe ansatz method for integrable models ${ }^{40-42,46-49}$ and the NRG method. An important aspect of the latter, allowing it to access thermodynamic properties on all temperature scales down to $T=0$, is the use of a logarithmic grid to represent the quasicontinuous spectrum $\omega \in[-D,+D]$ of the host system $H_{0}$. Thus $\omega \rightarrow \omega_{n}=$ $\pm D \Lambda^{-n}, n=0,1, \ldots$, where the parameter $\Lambda>1$ achieves a separation of the many energy scales in $H_{0}$ and thus in $H$ (see Sec. II). A large $\Lambda \gg 1$ allows calculations to reach low temperatures in fewer steps within the iterative diagonalization procedure of the NRG, and, in addition, a large $\Lambda \gg 1$ reduces the size of the truncation errors at each step in this procedure. ${ }^{24}$ However, for $\Lambda \gg 1$, specific heats (and also susceptibilities), calculated by using a standard logarithmic grid, exhibit discretization oscillations, especially at low temperatures. ${ }^{50}$ On the other hand, calculations at smaller $\Lambda \lesssim 3$, with less severe discretization oscillations, are more prone to truncation errors. In order to be able to carry out accurate calculations at all temperatures, using $\Lambda \gg 1$, an averaging over several discretizations of the host degrees of freedom has been introduced which essentially allows exact calculations to be carried out. ${ }^{50,51}$ With this refinement, the NRG approach has been used extensively in calculations of specific heats of quantum impurity models, ${ }^{39}$ with applications to the twoimpurity Kondo model ${ }^{52,53}$ and the two-channel Anderson models. ${ }^{54}$

The paper is organized as follows. In Sec. II the Anderson impurity model is described, and the NRG is outlined together with a brief description of how thermodynamic properties are conventionally calculated within NRG (at $\Lambda \gg 1$ ). In Sec. III we describe our new approach to specific heats of quantum impurity models using the Anderson impurity model as an example (with some further details given in Appendix A). The availability of exact Bethe ansatz results for this model ${ }^{40-42}$ allows a detailed evaluation of the accuracy of our new approach to specific heats. Results at zero and finite magnetic fields are presented in Sec. IV for the symmetric Anderson model. These are compared to both exact Bethe ansatz results and results obtained in the conventional NRG approach. Section V contains results for the asymmetric model with comparisons to corresponding Bethe ansatz calculations. The thermodynamic Bethe ansatz (TBA) equations for the Anderson impurity model and the details of their numerical solution can be found in Appendix B. In Sec. VI we present the generalization to multichannel and multiorbital Anderson impurity models and to dissipative two-state systems. For the Ohmic case, results for specific heats are compared to corresponding Bethe ansatz results for the equivalent anisotropic Kondo model (AKM). Section VII summarizes the main results of this paper and discusses possible future applications.

\section{MODEL, METHOD, AND CONVENTIONAL APPROACH TO THERMODYNAMICS}

We consider the Anderson impurity model,, 55 described by the Hamiltonian

$$
H=H_{\text {imp }}+H_{0}+H_{\text {int }} .
$$

The first term $H_{\mathrm{imp}}=\sum_{\sigma} \varepsilon_{d} d_{\sigma}^{\dagger} d_{\sigma}+U n_{d \uparrow} n_{d \downarrow}$ describes the impurity with local level energy $\varepsilon_{d}$ and on-site Coulomb repulsion $U$, the second term $H_{0}=\sum_{k \sigma} \epsilon_{k} c_{k \sigma}^{\dagger} c_{k \sigma}$ is the kinetic energy of noninteracting conduction electrons with dispersion $\varepsilon_{k}$, and the last term $H_{\text {int }}=\sum_{k \sigma} V_{k}\left(c_{k \sigma}^{\dagger} d_{\sigma}+d_{\sigma}^{\dagger} c_{k \sigma}\right)$ is the hybridization between the local level and the conduction electron states, with $V_{k}$ being the hybridization matrix element. We shall also consider the effect of a magnetic field of strength $B$ by adding a term $H_{B}=-g \mu_{\mathrm{B}} B S_{z}$ to $H$, where $S_{z}$ is the $z$ component of the total spin (i.e., impurity plus conduction electron spin), $g$ is the electron $g$ factor, and $\mu_{\mathrm{B}}$ is the Bohr magneton. We choose units such that $g=\mu_{\mathrm{B}}=1$.

The NRG procedure consists of the following steps. First, the conduction electron energies $-D \leqslant \varepsilon_{k} \leqslant D$, where $D$ is the half-bandwidth, are logarithmically discretized about the Fermi level $\varepsilon_{F}=0$, that is, $\epsilon_{k} \rightarrow \epsilon_{n}= \pm D \Lambda^{-n}, n=$ $0,1, \ldots$, where $\Lambda>1$ is a momentum rescaling factor. We shall also consider generalized discretizations defined by a parameter $z$, such that $\epsilon_{0}= \pm D$ and $\epsilon_{n}=$ $\pm D \Lambda^{-n-(1-z)}, n=1, \ldots$, with $z=1$ recovering the usual discretization. For $\Lambda \gg 1$, discretization induced oscillations of period $\ln \Lambda$ can be eliminated by averaging results for several $z$ in $(0,1] .^{50,51}$ Second, the operators $c_{n \sigma}, n=0,1, \ldots$ are rotated to a new set $f_{n \sigma}, n=0,1, \ldots$, with $V f_{0 \sigma}=\sum_{n=0}^{\infty} V_{k_{n}} c_{n \sigma}$, such that the discretized conduction band $H_{0}=\sum_{n=0 \sigma}^{\infty} \pm E_{n}(z) c_{n \sigma}^{\dagger} c_{n \sigma}$, with, for example, $E_{n}(z)=\frac{1}{2}\left(1+\Lambda^{-1}\right) D \Lambda^{-n}$ for $z=1$, takes the tridiagonal form $H_{0} \rightarrow \sum_{n=0 \sigma}^{\infty} \tilde{\epsilon}_{n}(z) f_{n \sigma}^{\dagger} f_{n \sigma}+\sum_{n=0 \sigma}^{\infty} t_{n}(z)\left(f_{n \sigma}^{\dagger} f_{n+1 \sigma}+\right.$ $\left.f_{n+1 \sigma}^{\dagger} f_{n \sigma}\right)$ in the new basis. Finally, within this new basis, the sequence of truncated Hamiltonians $H_{m}, m=0,1, \ldots$, where $H_{m}=H_{\mathrm{imp}}+H_{\mathrm{hyb}}+\sum_{n=0 \sigma}^{m} \tilde{\epsilon}_{n}(z) f_{n \sigma}^{\dagger} f_{n \sigma}+\sum_{n=0 \sigma}^{m-1}$ $t_{n}(z)\left(f_{n \sigma}^{\dagger} f_{n+1 \sigma}+f_{n+1 \sigma}^{\dagger} f_{n \sigma}\right)$, with $H_{\mathrm{hyb}}=V \sum_{\sigma}\left(f_{0 \sigma}^{\dagger} d_{\sigma}+\right.$ $\left.d_{\sigma}^{\dagger} f_{0 \sigma}\right)$, is iteratively diagonalized by using the recursion 
relation $H_{m+1}=H_{m}+\sum_{\sigma} \tilde{\epsilon}_{m+1}(z) f_{m+1 \sigma}^{\dagger} f_{m+1 \sigma}+\sum_{\sigma} t_{m}(z)$ $\left(f_{m \sigma}^{\dagger} f_{m+1 \sigma}+f_{m+1 \sigma}^{\dagger} f_{m \sigma}\right)$. This procedure ${ }^{24-26}$ yields the eigenstates $|p\rangle_{m}$ and eigenvalues $E_{p}^{m}$ on a decreasing set of energy scales $\omega_{m}(z) \sim t_{m}(z), m=0,1, \ldots$ Since the number of states increases as $4^{m+2}$, only the lowest states are retained for $m \geqslant m_{0}$, where typically $m_{0} \geqslant 4-5$. This is implemented either by (i) specifying an approximately constant number of states $N_{\text {keep }}$ to retain at each $m \geqslant m_{0}$, and $m_{0}$ will be fixed by the precise value of $N_{\text {keep }}$, or (ii) by specifying that only those states with rescaled energies $\left(E_{p}^{m}-E_{\mathrm{GS}}^{m}\right) / t_{m}(z)<e_{c}(\Lambda)$ be retained for $m \geqslant m_{0}$, for some predefined $m_{0}$, where $E_{\mathrm{GS}}^{m}$ is the (absolute) ground-state energy at iteration $m$ and $e_{c}(\Lambda)$ is $\Lambda$-dependent cut-off energy. Combining the information from all iterations then allows the calculation of thermodynamics on all temperature scales of interest. ${ }^{39,50}$ For most of the results in this paper we used the truncation scheme (ii) with $m_{0}=4-5$ and $e_{c}(\Lambda)=20 \sqrt{\Lambda}$, similar to the choice in Ref. 39. Some calculations using the truncation scheme (i) with $N_{\text {keep }}=860$ were also carried out in Sec. VI B. Both schemes were found to work well by comparison with exact Bethe ansatz calculations. Whereas in scheme (i) a fixed number $N_{\text {keep }}$ of levels is retained for all iterations $m \geqslant m_{0}$, in scheme (ii) the number of retained states, initially large for $m \lesssim m_{0}$ (typically several thousand), starts to decrease with increasing $m$, eventually saturating to a few hundred states at $m \gg m_{0}$ (e.g., for $\Lambda=4$ ). While in both schemes only the retained states of iteration $m$ are used to set up the Hamiltonian $H_{m+1}$ for the next iteration, all states of iteration $m$ are available, and are used, in practice, to calculate the thermodynamics.

The specific heat is calculated within the approach of Campo and Oliveira in Ref. 51, which we shall refer to as the "conventional" approach: For any temperature $T$ we choose the smallest $m$ such that $k_{\mathrm{B}} T>t_{m}(z)$ and we use the eigenvalues of $H_{m}$ to evaluate the partition function $Z_{m}(T)=$ $\sum_{p} e^{-E_{p}^{m} / k_{\mathrm{B}} T}$. The expectation value $\langle H\rangle$ is then calculated, followed by $\left\langle(H-\langle H\rangle)^{2}\right\rangle$ and the specific heat $C(T)$ [in addition, the thermodynamic potential $\Omega(T)=-k_{\mathrm{B}} T \ln Z_{m}(T)$ may also be calculated]. Calculations are carried out for several values of the $z$ parameter and then averaged. In the calculations reported below, we choose $z=(2 i-1) / 2 n_{z}, i=$ $1, \ldots, n_{z}$ with $n_{z}=2,4$, or 8 . This procedure is repeated for the conduction band Hamiltonian $H_{0}$ to obtain the host contribution to the specific heat $C_{0}(T)$. Finally, the impurity specific heat is obtained via $C_{\text {imp }}(T)=C(T)-C_{0}(T)$. The above prescription works well for $\Lambda \geqslant 4$ since the use of large $\Lambda$ reduces the size of truncation errors during the iterative diagonalization of $\mathrm{H}$ and $H_{0}{ }^{24}$ Furthermore, the use of large $\Lambda$ implies that the highest states of $H_{m}$ have energies $\gg t_{m}(z) \sim T$ so that $Z_{m}(T)$ is a good approximation to the partition function of the infinite system at temperature $T$. In addition to the specific heat, we also calculate the impurity contribution to the entropy $S_{\text {imp }}(T)=S(T)-S_{0}(T)$, where $S(T)$ and $S_{0}(T)$ are the entropies for $H$ and $H_{0}$, respectively, and

$$
\begin{gathered}
S(T)=-\frac{\partial \Omega}{\partial T}=k_{\mathrm{B}} \ln Z(T)+\langle H\rangle / T, \\
S_{0}(T)=-\frac{\partial \Omega_{0}}{\partial T}=k_{\mathrm{B}} \ln Z_{0}(T)+\left\langle H_{0}\right\rangle / T .
\end{gathered}
$$

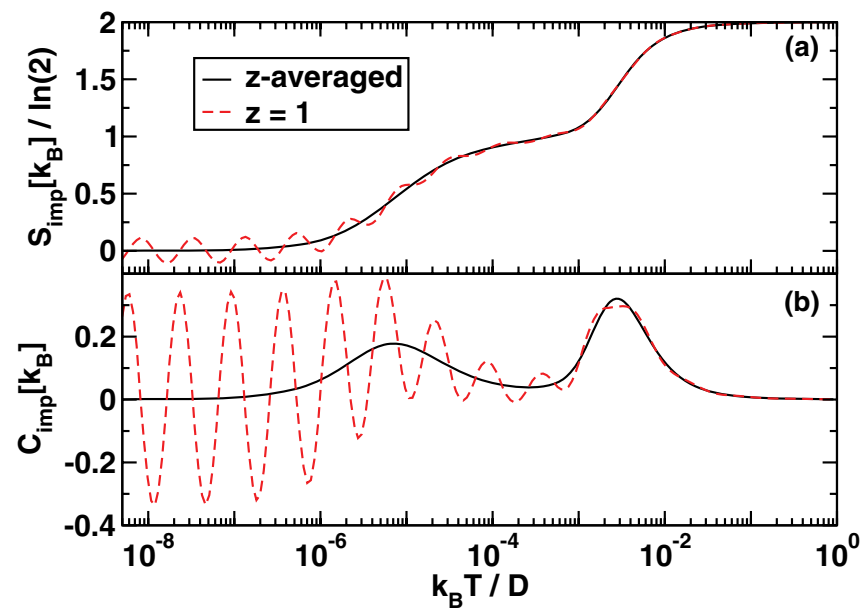

FIG. 1. (Color online) Temperature dependence of (a) the impurity entropy $S_{\text {imp }}(T)$, and (b) the impurity specific heat $C_{\text {imp }}(T)$ for the symmetric Anderson model with $U / \Delta_{0}=12$ and $\Delta_{0}=0.001 D$. The calculations are for $\Lambda=4$ with an energy cut-off $e_{c}(\Lambda=4)=40$, without $z$ averaging $\left[n_{z}=1, z=1\right.$ (dashed lines)], and with $z$ averaging $\left[n_{z}=2, z=1 / 4,3 / 4\right.$ (solid lines)]. For $\Lambda=4$ two $z$ values suffice to eliminate the discretization oscillations.

Unless otherwise specified, the NRG calculations presented in this paper will be for a band of half-width $D=1$ and a constant particle-hole symmetric density of states $N_{F}=$ $1 / 2 D$. The hybridization strength $\Delta_{0}$ defined as the half-width of the resonant level is given by $\Delta_{0}=\pi N_{F} V^{2}$. Calculations for the positive and negative- $U$ Anderson models include a $U(1)$ symmetry for total electron number conservation and SU(2) symmetry for total spin conservation. We use the discretization scheme of Campo and Oliveira in Ref. 51.

Figure 1 shows the temperature dependence of the specific heat and entropy, calculated with the above procedure, for the symmetric Anderson model with $U / \Delta_{0}=12$ and $\Delta_{0}=0.001 D$. The calculations are for $\Lambda=4$ using an energy cut-off $e_{c}(\Lambda=4)=40$, both without $z$ averaging $\left(n_{z}=1\right)$ and with $z$ averaging $\left(n_{z}=2\right)$. Note the aforementioned oscillations in the case of no $z$ averaging $\left(n_{z}=1\right)$. For $\Lambda=4$, two $z$ values suffice to eliminate the discretization oscillations (whereas for $\Lambda=10$, four values are required). In order to quantify the accuracy of the NRG calculations, we also solved numerically the thermodynamic Bethe ansatz equations for the Anderson model and calculated the entropy and specific heat (see Appendix B for details). A comparison of the $z$-averaged $\mathrm{NRG}$ calculations with the exact Bethe ansatz results, shown in Fig. 2, indicates very good agreement. Nevertheless, in the next section we show that the specific heat can be calculated directly from the impurity contribution to the internal energy in terms of local static correlation functions and that discretization effects within this approach are less pronounced than those above.

\section{IMPURITY INTERNAL ENERGY AND SPECIFIC HEATS}

The impurity internal energy is defined by $E_{\mathrm{imp}}=E_{\text {total }}-$ $E_{0}$, where $E_{\text {total }}=\langle H\rangle$ and $E_{0}=\left\langle H_{0}\right\rangle=\sum_{k \sigma} \epsilon_{k}\left\langle c_{k \sigma}^{\dagger} c_{k \sigma}\right\rangle_{0}$, where the subscript 0 denotes a thermodynamic average for noninteracting conduction electrons (i.e., impurity is absent). 


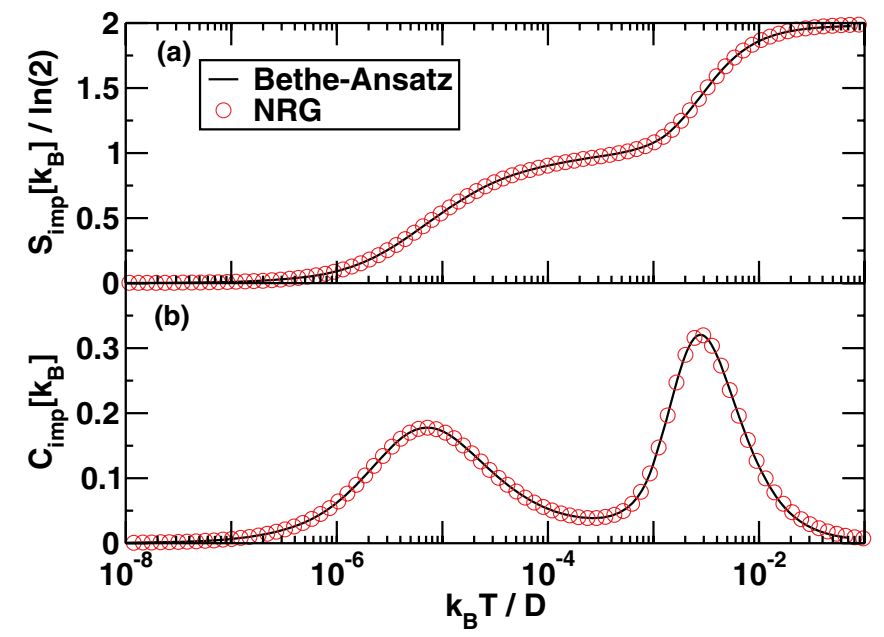

FIG. 2. (Color online) Temperature dependence of (a) the impurity entropy $S_{\text {imp }}(T)$ and (b) the impurity specific heat $C_{\text {imp }}(T)$ for the symmetric Anderson model with $U / \Delta_{0}=12$ and $\Delta_{0}=0.001 D$. Symbols: NRG calculations using the conventional approach. Solid lines: Bethe ansatz calculations. The NRG calculations are $z$ averaged with $n_{z}=2$ and other parameters as in Fig. 1.

We have

$$
E_{0}=\sum_{\sigma} \int d \epsilon f(\epsilon) \epsilon N(\epsilon),
$$

where $f(\epsilon)$ is the Fermi function and $N(\epsilon)=\sum_{k} \delta\left(\epsilon-\epsilon_{k}\right)$ is the noninteracting conduction electron density of states per spin. $E_{\text {total }}$ has four contributions:

$$
E_{\text {total }}=E_{\mathrm{occ}}+E_{\mathrm{docc}}+E_{\mathrm{cond}}+E_{\mathrm{hyb}},
$$

where $\quad E_{\text {occ }}=\sum_{\sigma} \varepsilon_{d}\left\langle n_{d \sigma}\right\rangle, \quad E_{\text {docc }}=U\left\langle n_{d \uparrow} n_{d \downarrow}\right\rangle, \quad E_{\text {cond }}=$ $\sum_{k \sigma} \epsilon_{k}\left\langle c_{k \sigma}^{\dagger} c_{k \sigma}\right\rangle$, and $E_{\mathrm{hyb}}=V \sum_{k \sigma}\left\langle c_{k \sigma}^{\dagger} d_{\sigma}+d_{\sigma}^{\dagger} c_{k \sigma}\right\rangle$. The first two contributions are evaluated as thermodynamic averages within the NRG calculation, requiring the calculation of matrix elements of $\sum_{\sigma} n_{d \sigma}$ and the double occupancy operator $\hat{D}_{\text {occ }}=n_{d \uparrow} n_{d \downarrow}$. The contribution $E_{\text {hyb }}$ may also be evaluated as a thermodynamic average $E_{\text {hyb }}=V \sum_{\sigma}\left\langle d_{\sigma}^{\dagger} f_{0 \sigma}+\right.$ H.c. $\rangle$. For the discussion below it is useful to note that the contribution $E_{\text {hyb }}$ can also be expressed in terms of the local retarded $d$-electron Green function $G_{d \sigma}(\omega)=\left\langle\left\langle d_{\sigma} ; d_{\sigma}^{\dagger}\right\rangle\right\rangle_{\omega+i \delta}$ and the hybridization function $\Delta(\omega)=\sum_{k} V^{2} /\left(\omega+i \delta-\epsilon_{k}\right)$ as

$$
E_{\mathrm{hyb}}=-\frac{2}{\pi} \sum_{\sigma} \int d \omega f(\omega) \operatorname{Im}\left[G_{d \sigma}(\omega) \Delta(\omega)\right] .
$$

Next, consider the contribution $E_{\text {cond }}=\sum_{k \sigma} \epsilon_{k}\left\langle c_{k \sigma}^{\dagger} c_{k \sigma}\right\rangle$. This is not simply $E_{0}$ since the impurity affects the conduction electrons once $V$ is finite. It can be evaluated from the equation of motion of the retarded conduction electron Greens function $G_{k \sigma}(\omega)=\left\langle\left\langle c_{k \sigma} ; c_{k \sigma}^{\dagger}\right\rangle\right\rangle_{\omega+i \delta}$ :

$$
G_{k \sigma}=G_{k \sigma}^{0}+G_{k \sigma}^{0} \mathcal{T}_{\sigma} G_{k \sigma}^{0} .
$$

Here $\mathcal{T}_{\sigma}(\omega)=V^{2} G_{d \sigma}(\omega)$ is the local T matrix and $G_{k \sigma}^{0}(\omega)=$ $1 /\left(\omega+i \delta-\epsilon_{k}\right)$ is the noninteracting conduction electron Greens function. Using

$$
\left\langle c_{k \sigma}^{\dagger} c_{k \sigma}\right\rangle=-\frac{1}{\pi} \int d \omega f(\omega) \operatorname{Im}\left(\left\langle\left\langle c_{k \sigma} ; c_{k \sigma}^{\dagger}\right\rangle\right)\right.
$$

we find for $E_{\text {cond }}$,

$$
E_{\text {cond }}=E_{0}+E_{\text {int }},
$$

where

$$
\begin{aligned}
E_{\mathrm{int}} & =-\frac{1}{\pi} \sum_{\sigma} \int d \omega f(\omega) \int d \epsilon \operatorname{Im}\left[\frac{\epsilon V^{2} N(\epsilon)}{(\omega+i \delta-\epsilon)^{2}} G_{d \sigma}(\omega)\right] \\
& =-\frac{1}{\pi} \sum_{\sigma} \int d \omega f(\omega) \operatorname{Im}\left[G_{d \sigma}(\omega) I(\omega)\right],
\end{aligned}
$$

where $I(\omega)$ is given by

$$
I(\omega)=-\frac{1}{\pi} \int d \epsilon \frac{\epsilon \Delta_{\mathrm{I}}(\epsilon)}{(\omega+i \delta-\epsilon)^{2}}=-\frac{\partial}{\partial \omega}[\omega \Delta(\omega)],
$$

with $\Delta_{\mathrm{I}}(\epsilon)=\operatorname{Im}[\Delta(\epsilon+i \delta)]=-\pi V^{2} N(\epsilon)$, and we evaluated $I(\omega)$ analytically by noting that $\Delta(\omega+i \delta)$ has the same properties as a retarded Green function (see Appendix A for details). We therefore find

$$
\begin{aligned}
E_{\text {int }} & =\frac{1}{\pi} \sum_{\sigma} \int d \omega f(\omega) \operatorname{Im}\left\{G_{d \sigma}(\omega) \frac{\partial}{\partial \omega}[\omega \Delta(\omega)]\right\} \\
& =E_{\text {int }}^{(1)}+E_{\text {int }}^{(2)},
\end{aligned}
$$

$$
\begin{aligned}
& E_{\mathrm{int}}^{(1)}=\frac{1}{\pi} \sum_{\sigma} \int d \omega f(\omega) \operatorname{Im}\left[G_{d \sigma}(\omega) \Delta(\omega)\right], \\
& E_{\mathrm{int}}^{(2)}=\frac{1}{\pi} \sum_{\sigma} \int d \omega f(\omega) \operatorname{Im}\left[G_{d \sigma}(\omega) \omega \frac{\partial \Delta(\omega)}{\partial \omega}\right] .
\end{aligned}
$$

From this and Eq. (9) we see that $E_{\text {int }}^{(1)}=-\frac{1}{2} E_{\text {hyb }}$. Hence, the impurity contribution to the internal energy $E_{\text {imp }}=E_{\text {total }}-E_{0}$ is given by

$$
\begin{aligned}
E_{\mathrm{imp}} & =E_{\mathrm{occ}}+E_{\mathrm{docc}}+\frac{1}{2} E_{\mathrm{hyb}}+E_{\mathrm{int}}^{(2)} \\
& =E_{\mathrm{ionic}}+\frac{1}{2} E_{\mathrm{hyb}}+E_{\mathrm{int}}^{(2)},
\end{aligned}
$$

where $E_{\text {ionic }}=\left\langle H_{\text {imp }}\right\rangle=E_{\text {occ }}+E_{\text {docc }}$ is adiabatically connected to the energy of the impurity decoupled from the band (i.e., its energy at $V \rightarrow 0$ ). All contributions to $E_{\mathrm{imp}}$, except for the last one, can be evaluated as thermodynamic averages of local static correlation functions: The contribution $E_{\text {int }}^{(1)}$ from the band which involves a finite frequency Greens function has been related to $E_{\text {hyb }}$, which can be evaluated as local static correlation function $V \sum_{\sigma}\left\langle d_{\sigma}^{\dagger} f_{0 \sigma}+\right.$ H.c. $\rangle$. The contribution $E_{\text {int }}^{(2)}$ also involves a finite frequency Greens function, but cannot be expressed as a local static correlation function. Its temperature dependence, however, is negligible since the main temperature dependence arises from the Fermi window $|\omega|<T$, but this region is cut out in $E_{\text {int }}^{(2)}$ due to the factor of $\omega$. In addition, for many cases of interest $\partial[\Delta(\omega)] / \partial \omega$ is small and vanishes in the wide band limit: $D \rightarrow \infty$ and $\Delta_{0}=\pi N(0) V^{2}$ fixed. For example, for a constant density of states it equals $\frac{2 \Delta_{0}}{\pi D}\left[1-(\omega / D)^{2}\right]^{-1} \sim \Delta_{0} / D$ for $\omega \ll D$. Thus, to a very good approximation, which we shall quantify in the rest of the paper with detailed numerical calculations and comparisons to exact Bethe ansatz results, we can approximate the impurity contribution to the specific heat and entropy via 


$$
\begin{aligned}
\bar{E}_{\mathrm{imp}}=E_{\mathrm{ionic}}+\frac{1}{2} E_{\mathrm{hyb}} \text { as } \\
C_{\mathrm{imp}}(T)=\frac{\partial \bar{E}_{\mathrm{imp}}}{\partial T}=\frac{\partial E_{\mathrm{occ}}}{\partial T}+\frac{\partial E_{\mathrm{docc}}}{\partial T}+\frac{1}{2} \frac{\partial E_{\mathrm{hyb}}}{\partial T} \\
=\frac{\partial E_{\mathrm{ionic}}}{\partial T}+\frac{1}{2} \frac{\partial E_{\mathrm{hyb}}}{\partial T}, \\
S_{\mathrm{imp}}(T)=\int_{0}^{T} d T^{\prime} \frac{C_{\mathrm{imp}}\left(T^{\prime}\right)}{T^{\prime}} .
\end{aligned}
$$

The omitted term $\partial E_{\text {int }}^{(2)} / \partial T$ in (15), as argued above, has a negligible temperature dependence (although its magnitude is not necessarily always small compared to the terms retained). Notice that $\bar{E}_{\text {imp }}$ is made up of a term due to the partial occupation of the local resonant level $\left(E_{\text {occ }}\right)$, a term due to the Coulomb repulsion of electrons in this level $\left(E_{\text {docc }}\right)$, and a term due to the energy gained by hybridization of the local level with the conduction electrons $\left(E_{\mathrm{hyb}} / 2\right)$, that is, it involves only local static correlation functions. Such quantities can be calculated very accurately and efficiently within the NRG method, within a single calculation for the total system only, a significant advantage of this approach. In some situations, the hybridization function $\Delta(\omega)$ may be strongly asymmetric and have a strong energy dependence close to $\omega=0$. In such cases, the term $E_{\text {int }}^{(2)}$ can be calculated via the local spectral function and included in $E_{\mathrm{imp}}$, which is possible within the $\mathrm{NRG}$, at somewhat higher numerical cost. Another advantage of the present approach is that discretization oscillations are far smaller for local quantities appearing in $\bar{E}_{\text {imp }}$ than for extensive quantities, such as $\langle H\rangle$ and $\left\langle(H-\langle H\rangle)^{2}\right\rangle$ appearing in the conventional approach to specific heats. Figure 3 shows the specific heat and entropy calculated with the above method for the same parameters as in Figs. 1 and 2, with and without $z$ averaging. One sees that the discretization oscillations in the case of no $z$ averaging $\left(n_{z}=1\right.$ curves) are drastically

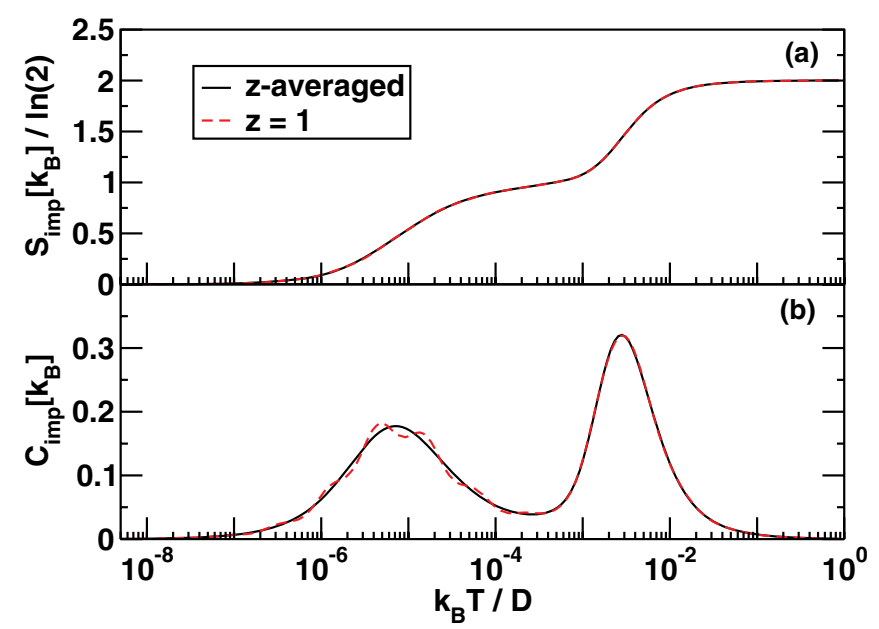

FIG. 3. (Color online) Temperature dependence of (a) the impurity entropy $S_{\mathrm{imp}}(T)$ and (b) the impurity specific heat $C_{\mathrm{imp}}(T)$ for the symmetric Anderson model with $U / \Delta_{0}=12$ and $\Delta_{0}=0.001 D$ calculated within NRG using the new approach for $\Lambda=4$ with an energy cutoff $e_{c}(\Lambda=4)=40$. Solid lines: $n_{z}=2$ ( $z$ averaging). Dashed lines: $n_{z}=1$ (no $z$ averaging). For $\Lambda=4$ two $z$ values thus suffice to eliminate the discretization oscillations at $n_{z}=1$.

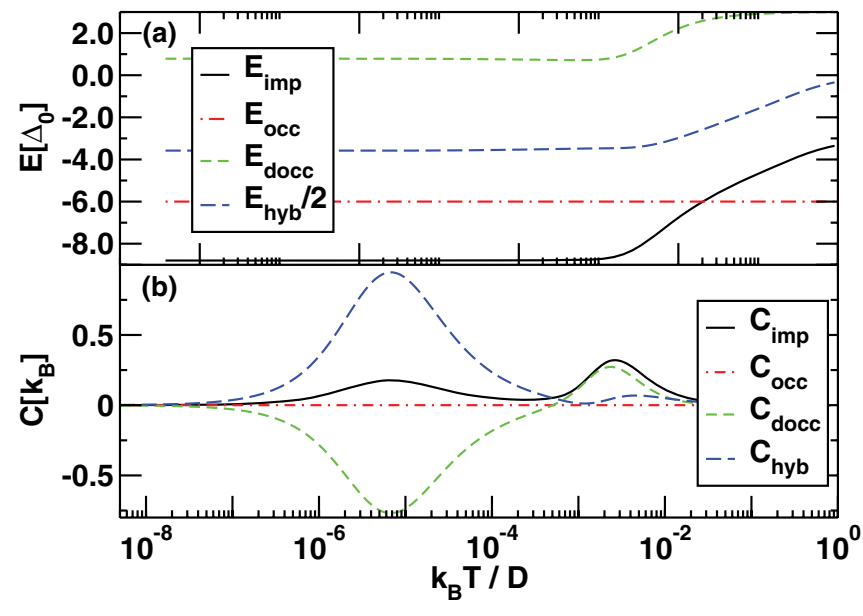

FIG. 4. (Color online) (a) The individual contributions $E_{\text {occ }}$, $E_{\text {docc }}$, and $E_{\text {hyb }}$ to $E_{\text {imp }}$ as a function of temperature (in units of $\left.\Delta_{0}\right)$ for the symmetric model with parameters as in Fig. $1(z$ averaged with $n_{z}=2$ ). (b) Temperature derivatives of the above, yielding the relative contributions $C_{\mathrm{occ}}, C_{\mathrm{docc}}$, and $C_{\mathrm{hyb}}$ to the specific heat $C_{\text {imp }}$.

smaller than for the corresponding $n_{z}=1$ results from the conventional approach in Fig. 1. Including $z$ averaging makes the results of the new procedure indistinguishable from the Bethe ansatz calculations, as will be discussed in detail in Secs. IV and V.

In Fig. 4(a) we show the different contributions $E_{\text {occ }}, E_{\text {docc }}$, and $E_{\mathrm{hyb}} / 2$ to the impurity internal energy for the symmetric Anderson model. Their temperature derivatives $C_{\mathrm{occ}}, C_{\mathrm{docc}}$, and $C_{\text {hyb }}$ give the relative contributions of these terms to the impurity specific heat $C_{\text {imp }}$ and are shown in Fig. 4(b). Notice that the Kondo induced peak in $C_{\mathrm{imp}}$ at low temperatures results from a delicate balance of the hybridization $\left(C_{\mathrm{hyb}}\right)$ and Coulomb contributions $\left(C_{\text {docc }}\right)$, while the peak due to the resonant level at high temperatures is mainly due to the Coulomb term. The latter trend persists also for the asymmetric model, as shown in Fig. 5. Notice also that the gain in energy due to hybridization diminishes at high temperatures, reflecting the decoupling of the impurity from the conduction electrons in this limit. In general, however, the interaction of the impurity with the environment via the hybridization term provides an essential contribution at all nonzero hybridization strengths.

We now quantify the error in neglecting $\partial E_{\text {int }}^{(2)}(T) / \partial T$ in Eq. (15) for the calculation of impurity specific heats by (a) comparing the result for $C_{\text {imp }}$ obtained within the new method with the Bethe ansatz calculations and (b) explicitly calculating the contribution $\partial E_{\text {int }}^{(2)}(T) / \partial T$. Figure 6(a) shows the comparison to the Bethe ansatz calculation, where we also include the specific heat from the conventional approach. The relative deviation of the NRG calculations to the Bethe ansatz, shown in Fig. 6(b), is below $1 \%$ for all temperatures $T<0.01=10 \Delta_{0}$. For $T \ll T_{\mathrm{K}}$, the relative error in $C_{\text {imp }}$ from the internal energy is $0.1 \%$ and $0.5 \%$ in the conventional approach. The relative error exhibits remnants of the discretization oscillations, which are not completely eliminated with $z$ averaging. Notice also that the errors in the two NRG calculations have the same error (relative to the Bethe ansatz) in the high-temperature 


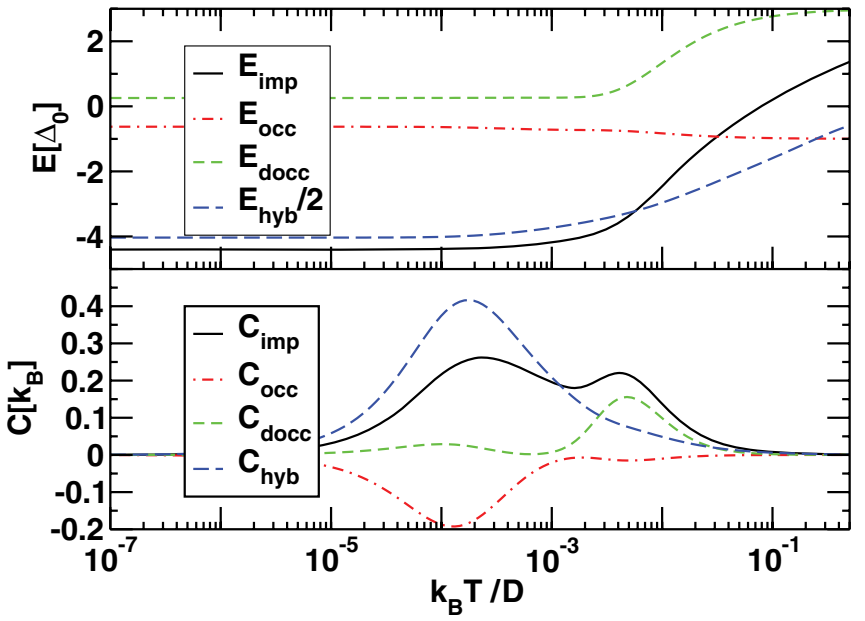

FIG. 5. (Color online) (a) The individual contributions $E_{\text {occ }}$, $E_{\text {docc }}$, and $E_{\text {hyb }}$ to $E_{\text {imp }}$ as a function of temperature (in units of $\Delta_{0}$ ) for the asymmetric model with parameters as in Fig. 1, but for an asymmetric level position $\varepsilon_{d} / \Delta_{0}=-1$ ( $z$ averaged with $\left.n_{z}=2\right)$. (b) Temperature derivatives of the above, yielding the relative contributions $C_{\mathrm{occ}}, C_{\mathrm{docc}}$, and $C_{\mathrm{hyb}}$ to the specific heat $C_{\mathrm{imp}}$.

limit $T \gg \Delta_{0}$. Hence, the latter error is not due to neglect of $E_{\text {int }}^{(2)}$ in Eq. (14). Instead, it reflects (a) the different high-energy cut-off schemes in NRG and Bethe ansatz and (b) the finite size errors in the high-energy excitation spectrum in NRG since the latter stem from the shortest chains diagonalized (typically $m=4-6$ ), which are also the ones most sensitive to the logarithmic discretization. The fact that the errors in both NRG calculations also correlate at lower temperatures ( $T \lesssim \Delta_{0}$ ) suggests that the neglect of $E_{\text {int }}^{(2)}$ in Eq. (14) is not the main source of error in calculating $C_{\text {imp }}(T)$. An explicit calculation that illustrates this is shown in Fig. 7. As stated above, the value of $E_{\text {int }}^{(2)}$ is of order $\Delta_{0} / \pi$, however, one clearly

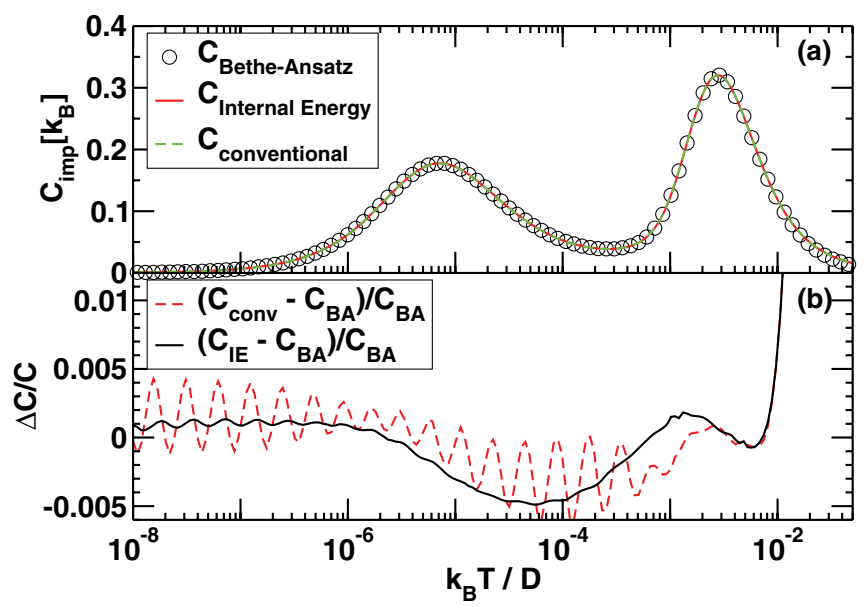

FIG. 6. (Color online) (a) Comparison of specific heat $C_{\text {imp }}(T)$ from the impurity internal energy (solid line) and conventional approach (dashed line) with the Bethe ansatz calculation (symbols) for the symmetric Anderson model with parameters as in Fig. 1. NRG parameters also as in Fig. 1 with $n_{z}=2$. (b) The relative deviation with respect to the Bethe ansatz result of the new (solid line) and conventional (dashed line) approaches.

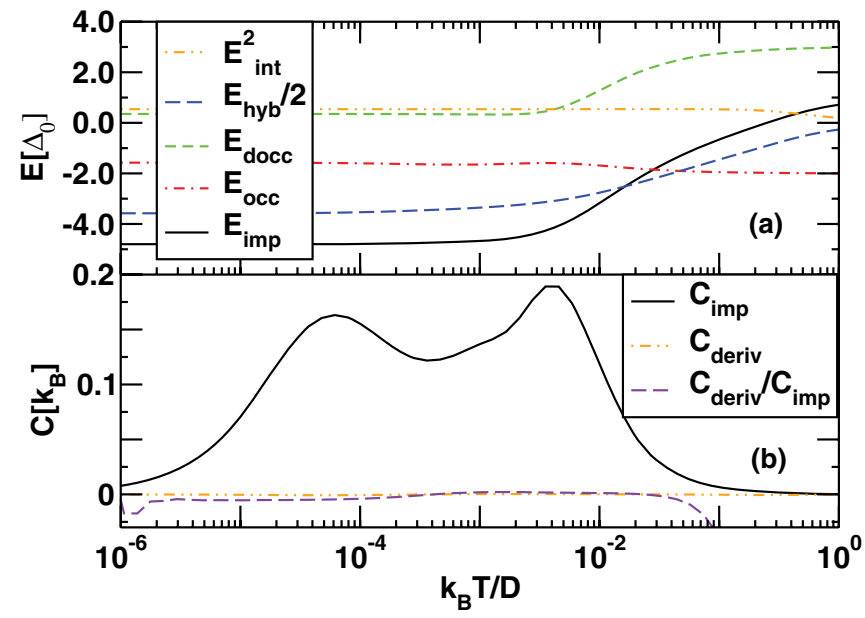

FIG. 7. (Color online) (a) The contribution $E_{\text {int }}^{(2)}$ to $E_{\text {imp }}$ as a function of temperature compared with $E_{\text {occ }}, E_{\text {docc }}$, and $E_{\text {hyb }}$ (in units of $\Delta_{0}$ ) for the asymmetric model. Model parameters: $U=12 \Delta_{0}, \Delta_{0}=0.001 D, \varepsilon_{d} / \Delta_{0}=-2$ with a semi-elliptic hybridization function $\operatorname{Im}[\Delta(\omega)]=-\frac{\Delta_{0}}{D} \sqrt{\left(D^{2}-\omega^{2}\right)}$. A small $\Lambda=$ 1.5 was used, which allows the spectral function entering $E_{\text {int }}^{(2)}$ to be obtained without $z$ averaging. (b) The contribution $C_{\text {deriv }}=$ $\partial E_{\text {int }}^{(2)}(T) / \partial T$ to $C_{\text {imp }}$. The relative size of $C_{\text {deriv }}$ to $C_{\text {imp }}$ lies between $0.2 \%$ and $0.5 \%$ for all temperatures, except at temperatures approaching the bandwidth $D=1$.

sees in Fig. 7(a) that $E_{\text {int }}^{(2)}$ has little temperature dependence (relative to the other contributions) for all temperatures extending up to the bandwidth $D=1$. Its relative contribution to the impurity specific heat, shown in Fig. 7(b), for an energy dependent $\Delta(\omega)$, is negligible, typically contributing below $0.5 \%$.

\section{RESULTS FOR THE SYMMETRIC MODEL}

In this section we show results for the entropy and specific heat of the Anderson model at the particle-hole symmetric point $\varepsilon_{d}=-U / 2$. Results for zero magnetic field and increasing correlation strength $U / \Delta_{0}$ are presented in Sec. IV A and results for finite magnetic fields are given in Sec. IV B.

The symmetric Anderson model has been investigated in detail $^{1}$ and is well understood. For $U / \Delta_{0} \gg 1$ and $-\varepsilon_{d} \gg \Delta_{0}$, a local spin $S=1 / 2$ magnetic moment forms on the impurity. In this limit, the physics of the symmetric model at low temperatures $T \ll \min \left(\left|\varepsilon_{d}+U\right|,\left|\varepsilon_{d}\right|, D\right)$ is that of the Kondo model

$$
H_{\mathrm{K}}=H_{0}+J \mathbf{S} \cdot \mathbf{s}_{0},
$$

where $J$ is an antiferromagnetic exchange coupling between the local spin $S$ and the conduction electron spin-density $\mathbf{s}_{0}$ at the impurity site. The value of $J$ is given by the SchriefferWolff transformation ${ }^{56} J=4 V^{2} / U$. The low-temperature properties (for $U \gg \Delta_{0}$ ) are universal functions of $T / T_{\mathrm{K}}$ and $B / T_{\mathrm{K}}$ where we choose to define the Kondo scale from the Bethe ansatz result for the $T=0$ susceptibility $\chi(0)$ via $\chi(0)=\left(g \mu_{\mathrm{B}}\right)^{2} / 4 T_{\mathrm{K}}$. For $U \gg \Delta_{0}, T_{\mathrm{K}}$ is given by

$$
T_{\mathrm{K}}=\sqrt{U \Delta_{0} / 2} e^{-\pi U / 8 \Delta_{0}+\pi \Delta_{0} / 2 U},
$$




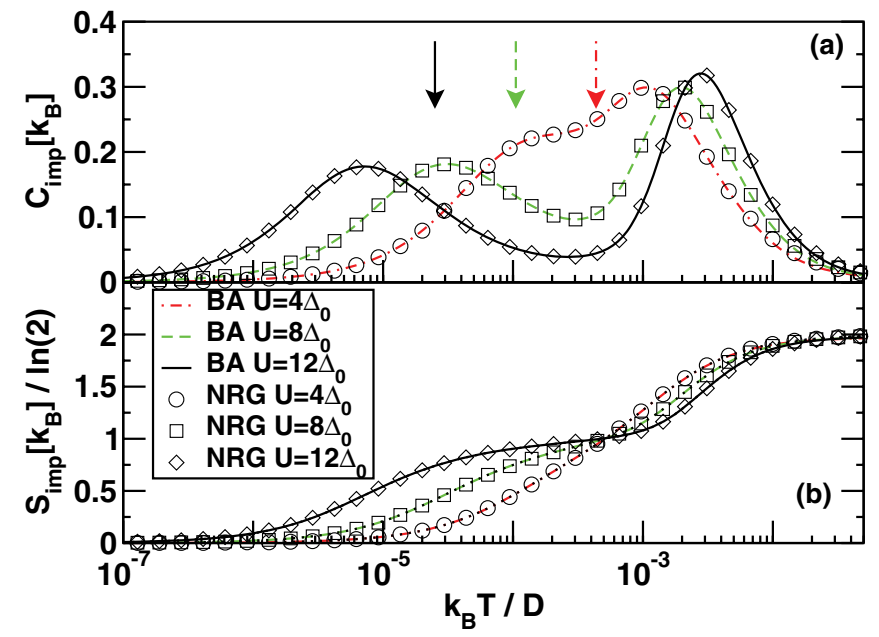

FIG. 8. (Color online) Temperature dependence of (a) the impurity specific heat $C_{\text {imp }}(T)$ and (b) the impurity entropy $S_{\text {imp }}(T) / \ln (2)$ for the symmetric Anderson model with $\Delta_{0}=0.001 \mathrm{D}$ and increasing values of the Coulomb interaction: $U / \Delta_{0}=4,8,12$. Arrows in (a) indicated the Kondo scale $T_{\mathrm{K}}$ defined in Eq. (18). Symbols: new approach using NRG with $\Lambda=4$ with an energy cutoff $e_{c}(\Lambda=4)=$ 40 , and $z$ averaging $\left[n_{z}=2, z=1 / 4,3 / 4\right]$. Lines: corresponding Bethe ansatz calculations.

within corrections which are exponentially small in $U / \pi \Delta_{0}$ (see Ref. 1). For $U=0$, the symmetric Anderson model reduces to a resonant level model and the relevant lowtemperature scale is then $\Delta_{0}$.

\section{A. Zero magnetic field}

A comparison of the new approach with Bethe ansatz calculations is shown in Fig. 8 for the temperature dependence of the impurity specific heat and entropy for increasing values of the Coulomb interaction $U / \Delta_{0}$. For $U / \Delta_{0}=12$, the Kondo induced peak in the specific heat at $T_{\mathrm{p}}=\alpha T_{\mathrm{K}}$ with $\alpha \approx 0.29$ is well separated from the peak at $T \approx\left|\varepsilon_{d}\right|$ due to the resonant level. With decreasing $U / \Delta_{0}$, the Kondo effect is suppressed and the Kondo induced peak in $C(T)$ eventually merges with the peak due to the resonant level for $U / \Delta_{0} \rightarrow 0$. Good agreement between the NRG and the exact Bethe ansatz calculations is seen for all values of $U / \Delta_{0}$.

\section{B. Finite magnetic field}

At finite magnetic fields $B>0$, the $\mathrm{SU}(2)$ spin symmetry which we use in the NRG calculations, is broken. Therefore, in order to carry out calculations at finite magnetic field $B>0$, preserving the numerical advantages of the full SU(2) symmetry, such as the increased number of states that can be retained, we obtained the finite field results by mapping the symmetric positive- $U$ Anderson model onto the negative- $U$ Anderson model in the absence of a magnetic field but with local level given by $\varepsilon_{d}=-U / 2-B / 2$ with $U$ negative. ${ }^{57,58}$ This correspondence results from a particle-hole transformation on the down spins only: $d_{\downarrow} \rightarrow d_{\downarrow}^{\dagger}, d_{\uparrow} \rightarrow d_{\uparrow}$, and $c_{k \downarrow} \rightarrow c_{-k \downarrow}^{\dagger}, c_{k \uparrow} \rightarrow c_{k \uparrow}$ with a particle-hole symmetric band $\epsilon_{k}=-\epsilon_{-k}$.

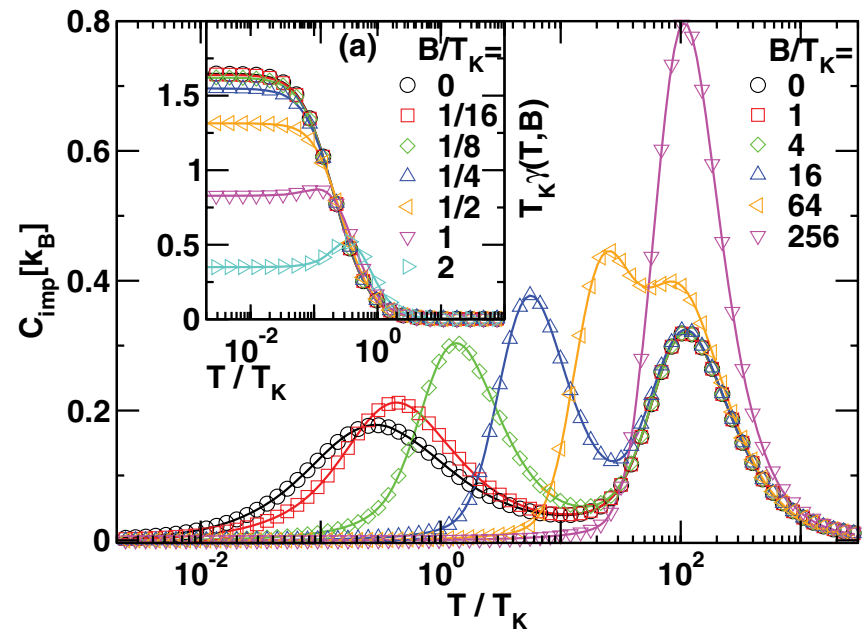

FIG. 9. (Color online) Temperature dependence of the impurity specific heat $C_{\text {imp }}(T, B)$ for the symmetric Anderson model for $U / \Delta_{0}=12, \Delta_{0}=0.001 D$, and increasing values of the magnetic field $B / T_{\mathrm{K}} \geqslant 1$, where the Kondo scale $T$ is defined in Eq. (18). Symbols: NRG calculations $\Lambda=4$ with an energy cut-off $e_{c}(\Lambda=4)=40$, and $z$ averaging $\left[n_{z}=2, z=1 / 4,3 / 4\right]$. Lines: Bethe ansatz calculations. Inset (a): $T_{\mathrm{K}} \gamma(T, B)$ versus $T / T_{\mathrm{K}}$ for several values of $B / T_{\mathrm{K}} \leqslant 2$, where $\gamma(T, B)=C_{\mathrm{imp}}(T, B) / T$.

Figure 9 shows the temperature dependence of $C_{\text {imp }}(T, B)$ for $B / T_{\mathrm{K}} \geqslant 1$ using our new approach and compared with Bethe ansatz calculations. The Kondo peak in the specific heat shifts to higher fields with increasing $B$ and its position scales as $B^{2} / T_{\mathrm{K}}$ for $T_{\mathrm{K}} \ll B \ll \varepsilon_{d}$. In contrast, the resonant level peak remains approximately fixed at $T \approx \varepsilon_{d}$. As $B$ approaches the value $\varepsilon_{d}$, the two peaks merge into one peak at $T \approx \varepsilon_{d}$, with approximately twice the height of the $B=0$ resonant level peak, and containing the whole entropy $S_{\text {imp }} / k_{\mathrm{B}}=\ln (4)$. The low-field behavior of $C_{\text {imp }}(T, B)$, also compared to Bethe ansatz calculations, is shown in Fig. 9(a) as $T_{\mathrm{K}} \gamma(T, B)=C_{\mathrm{imp}}(T, B) /\left(T / T_{\mathrm{K}}\right)$ versus $T / T_{\mathrm{K}}$ for $B / T_{\mathrm{K}} \leqslant 2$. For $T, B \rightarrow 0, \gamma(T, B) \rightarrow \gamma(0,0) \sim 1 / T_{\mathrm{K}}$, where $\gamma(0,0)$ is the linear coefficient of specific heat. This is strongly enhanced for $U / \Delta_{0} \gg 1$ due to the exponential decrease of $T_{\mathrm{K}}$. A finite magnetic field of order $T_{\mathrm{K}}$ significantly suppresses the Kondo effect and results in smaller values of $\gamma(0, B)$. As another check on the accuracy of our calculations, we estimate the Wilson ratio $R_{\mathrm{W}}=4 \pi^{2} \chi(0) / 3 \gamma(0,0)$. This takes the value 2 in the Kondo regime of the symmetric Kondo model (i.e., for $U \gg \Delta_{0}$ ). From the definition of $T_{\mathrm{K}}$ we have that the susceptibility $\chi(0)=1 / 4 T_{\mathrm{K}}$, and from Fig. 9(a) we extract $\gamma(0,0) \approx 1.64 / T_{\mathrm{K}}$, resulting in $R_{\mathrm{W}} \approx 2.006$, that is, a relative error in $R_{\mathrm{W}}$ below $1 \%$.

\section{RESULTS FOR THE ASYMMETRIC MODEL}

Figure 10 shows the impurity specific heat versus temperature for the asymmetric Anderson model, that is, for $\varepsilon_{d}>$ $-U / 2$, calculated within the new approach. For comparison we also show the corresponding Bethe ansatz calculations. One sees again excellent agreement at all temperatures between the two methods. Results for $\varepsilon_{d}<-U / 2$ are not shown since these can be obtained from results for $\varepsilon_{d}>-U / 2$ by 


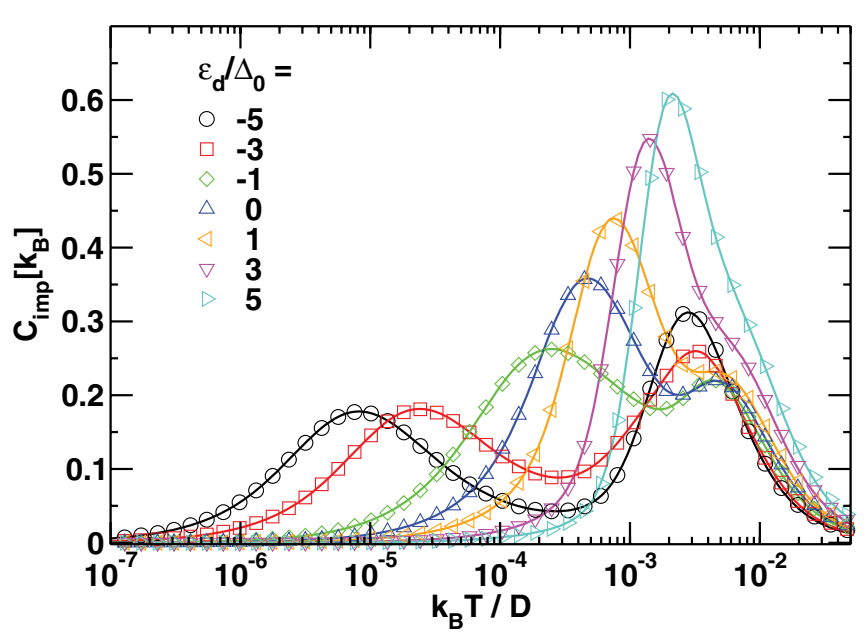

FIG. 10. (Color online) Temperature dependence of $C_{\text {imp }}(T)$ for $U / \Delta_{0}=12, \Delta_{0}=0.001 D$, and local level positions $\varepsilon_{d} / \Delta_{0}$ ranging from Kondo $\left(\varepsilon_{d} / \Delta_{0} \leqslant-1\right)$, mixed valence $\left(\left|\varepsilon_{d} / \Delta_{0}\right| \leqslant 1\right)$, and empty orbital $\left(\varepsilon_{d} / \Delta_{0}>1\right)$ regimes. Symbols: NRG calculations (new approach, $z$ averaging, and NRG parameters as in Fig. 1). Lines: Bethe ansatz calculations.

noting that the Anderson model with parameters $\varepsilon_{d}, U, V$ transforms, under a particle-hole transformation applied to both spin species, to an Anderson model with parameters $-\left(\varepsilon_{d}+U\right), U, V$. This holds for a particle-hole symmetric constant density of states, the case considered here.

The specific heat curves for the asymmetric model are more complicated than those of the symmetric model. In the latter, the relevant excitations were the low-temperature spin flip excitations, characterized by the Kondo scale $T_{\mathrm{K}}$, and the excitations involving addition or removal of an electron from the resonant level, both characterized by an energy $\left|\varepsilon_{d}\right|=U / 2$. This accounts for the two peaks in the specific heat of the symmetric model: A high-temperature peak at $T \approx\left|\varepsilon_{d}\right|$ and a low-temperature Kondo induced peak at $T \approx T_{\mathrm{K}}$. For the asymmetric Anderson model, three types of excitation are possible: Low-temperature spin flip excitations, associated with the Kondo scale $T_{\mathrm{L}}=\sqrt{U \Delta_{0} / 2} e^{-\pi\left|\varepsilon_{d}\right|\left|\varepsilon_{d}+U\right| / 2 U \Delta_{0}}$ of the asymmetric model, ${ }^{1}$ and excitations associated with (i) removing an electron from a singly occupied level (with energy scale $\left|\varepsilon_{d}\right|$ ) and (ii) removing an electron from a doubly occupied level (with energy scale $\left|\varepsilon_{d}+U\right|$ ). Thus, three peaks can be present in $C_{\text {imp }}(T)$ : A Kondo induced peak at $T \approx T_{\mathrm{L}}$, and two charge fluctuation induced peaks at $T \approx T_{1}=\left|\varepsilon_{d}\right|$ and $T \approx T_{2}=\left|\varepsilon_{d}+U\right|$, respectively. In Fig. 10 the two hightemperature peaks are seen in the mixed valence regime and partly also in the empty orbital regime (where the upper peak at $T_{2}$ appears as a shoulder of the main peak at $T_{1}$ ). However, in the Kondo regime, the cases $\varepsilon_{d} / \Delta_{0}=-5,-3$ with the choice $U=12 \Delta_{0}$ result in $T_{1} / \Delta_{0}=5,3$ and $T_{2} / \Delta_{0}=7,9$. In these cases, $T_{1}$ and $T_{2}$ are too close for separate peaks to be seen. In order to clarify this, we carried out calculations for $U=48 \Delta_{0} \gg \Delta_{0}$ and $\varepsilon_{d} / \Delta_{0}=-10,-8,-6,-4,-2$ in the Kondo regime, for which $T_{1} / \Delta_{0}=10,8,6,4,2$ and $T_{2} / \Delta_{0}=$ $38,40,42,44,46 \gg T_{1} / \Delta_{0}$ are disparate scales. Figure 11 shows how the peaks at $T \approx T_{1}$ and $T \approx T_{2}$ evolve from the peak at $T \approx\left|\varepsilon_{d}\right|=U / 2$ of the symmetric model (dashed line in Fig. 11) on increasing $\varepsilon_{d}$ above $-U / 2$. Simultaneously,

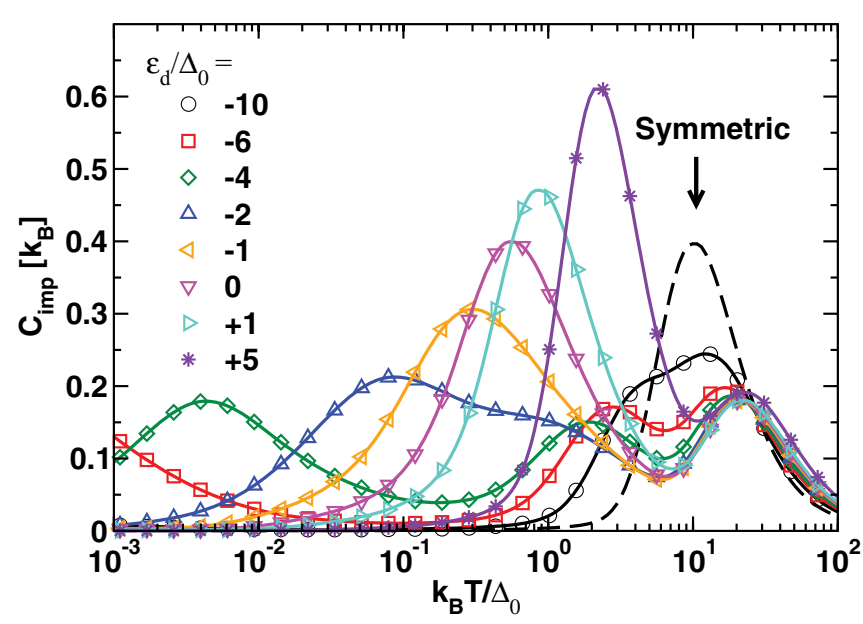

FIG. 11. (Color online) Temperature dependence of $C_{\text {imp }}(T)$ for $U / \Delta_{0}=48, \Delta_{0}=0.0001 D$, and local level positions $\varepsilon_{d} / \Delta_{0}=-10$, $-6,-4,-2$ (Kondo regime), $\varepsilon_{d} / \Delta_{0}=-1,0,+1$ (mixed valence regime), and $\varepsilon_{d} / \Delta_{0}=+5$ (empty orbital regime). NRG using the new approach (symbols) and conventional approach (solid lines) $[\Lambda=$ $\left.20, n_{z}=4, e_{c}(\Lambda)=130\right]$. Dashed line: resonant level peak in $C_{\mathrm{imp}}$ at $T / \Delta_{0} \approx\left|\varepsilon_{d}\right| / \Delta_{0}=U / 2 \Delta_{0}=24$ for the symmetric model (the Kondo induced peak at much lower $T$ is not shown). The two hightemperature peaks of the asymmetric model evolve from this peak when the asymmetry is finite.

the Kondo peak in the specific heat at $T_{\mathrm{L}}$ shifts to higher temperatures and eventually merges with the peak at $T_{1}$ when the mixed valence regime is reached (i.e., for $\varepsilon_{d}=-\Delta_{0}$ ). Thereafter, only the high-temperature peaks at $T_{1}$ and $T_{2}$ are present. Notice also that in the mixed valence regime $T_{1}$ differs significantly from $\left|\varepsilon_{d}\right|$, a result of nontrivial renormalizations present in the mixed valence regime, but absent in the empty orbital regime.

\section{GENERALIZATION TO OTHER MODELS}

The approach of Sec. III can be straightforwardly generalized to multiorbital and multichannel Anderson impurity models with arbitrary local Coulomb interactions, as we briefly outline in Sec. VI A. In addition, in Sec. VIB we discuss its application to dissipative two-state systems and the anisotropic Kondo model (AKM).

\section{A. Multiorbital and multichannel Anderson models}

The multiorbital and multichannel Anderson impurity model is given by $H=H_{\text {imp }}+H_{0}+H_{\text {int }}$, where $H_{\text {imp }}=$ $\sum_{\alpha \sigma} \varepsilon_{\alpha} d_{\alpha \sigma}^{\dagger} d_{\alpha \sigma}+H_{\mathrm{C}}\left(U, U^{\prime}, J\right)$ describes the impurity with a set of local levels having energies $\varepsilon_{d \alpha}, \alpha=1, \ldots, g$ and $H_{\mathrm{C}}\left(U, U^{\prime}, J\right)$ is the local Coulomb interaction involving intraorbital $U$, interorbital $U^{\prime}$, and a Hund's exchange term $J$. The conduction electrons are described by $H_{0}=\sum_{k \alpha \sigma} \epsilon_{k \alpha} c_{k \alpha \sigma}^{\dagger} c_{k \alpha \sigma}$, where $\epsilon_{k \alpha}$ is the kinetic energy of electrons in band $\alpha$. These bands hybridize with hybridization strengths $V_{\alpha}, \alpha=1, \ldots, g$ to the local levels via $H_{\text {int }}=\sum_{k \alpha \sigma} V_{\alpha}\left(c_{k \alpha \sigma}^{\dagger} d_{\alpha \sigma}+d_{\alpha \sigma}^{\dagger} c_{k \alpha \sigma}\right)$. Let $\Delta_{\alpha}(\omega)=$ $\sum_{k} V_{\alpha}^{2} /\left(\omega-\epsilon_{k \alpha}\right)$ denote the hybridization functions characterizing $H_{\text {int }}$. Proceeding as in Sec. III, we write the impurity 
internal energy as $E_{\mathrm{imp}}=E_{\text {total }}-E_{0}$, where $E_{\text {total }}=\langle H\rangle$ is the total energy and $E_{0}=\left\langle H_{0}\right\rangle=\sum_{k \alpha \sigma} \epsilon_{k \alpha}\left\langle c_{k \alpha \sigma}^{\dagger} c_{k \alpha \sigma}\right\rangle_{0}$ is the energy of the noninteracting conduction electrons in the absence of the impurity. The latter is given by $E_{0}=$ $\sum_{\alpha \sigma} \int d \epsilon f(\epsilon) \epsilon N_{\alpha}(\epsilon)$, where $f(\epsilon)$ is the Fermi function and $N_{\alpha}(\epsilon)=\sum_{k} \delta\left(\epsilon-\epsilon_{k \alpha}\right)$ is the noninteracting conduction electron density of states per spin for band $\alpha$. $E_{\text {total }}$ is a sum of local occupation number contributions $E_{\text {occ }}=$ $\sum_{\alpha \sigma}\left\langle\varepsilon_{\alpha} n_{\alpha \sigma}\right\rangle$ and local Coulomb terms $E_{C}=\left\langle H_{C}\left(U, U^{\prime}, J\right)\right\rangle$ and two further terms involving the interacting band $E_{\text {cond }}=$ $\sum_{k \alpha \sigma} \epsilon_{k \alpha}\left\langle c_{k \alpha \sigma}^{\dagger} c_{k \alpha \sigma}\right\rangle$ and the hybridization energy $E_{\mathrm{hyb}}=$ $\sum_{\alpha \sigma} V_{\alpha}\left\langle d_{\alpha \sigma}^{\dagger} f_{0 \alpha \sigma}+\right.$ H.c. $\rangle$, where $V_{\alpha} f_{0 \alpha \sigma}=\sum_{k} c_{k \alpha \sigma}$ :

$$
E_{\mathrm{total}}=E_{\mathrm{occ}}+E_{\mathrm{C}}+E_{\mathrm{cond}}+E_{\mathrm{hyb}}
$$

We evaluate the latter two contributions as in Sec. III, finding

$$
E_{\mathrm{hyb}}=-\frac{2}{\pi} \sum_{\alpha \sigma} \int d \omega f(\omega) \operatorname{Im}\left[G_{d \alpha \sigma}(\omega) \Delta_{\alpha}(\omega)\right],
$$

and $E_{\text {cond }}=E_{0}+E_{\text {int }}$, where

$$
\begin{aligned}
E_{\text {int }} & =\frac{1}{\pi} \sum_{\alpha \sigma} \int d \omega f(\omega) \operatorname{Im}\left\{G_{d \alpha \sigma}(\omega) \frac{\partial}{\partial \omega}\left[\omega \Delta_{\alpha}(\omega)\right]\right\} \\
& =E_{\text {int }}^{(1)}+E_{\text {int }}^{(2)}, \\
E_{\text {int }}^{(1)} & =\frac{1}{\pi} \sum_{\alpha \sigma} \int d \omega f(\omega) \operatorname{Im}\left[G_{d \alpha \sigma}(\omega) \Delta_{\alpha}(\omega)\right], \\
E_{\text {int }}^{(2)} & =\frac{1}{\pi} \sum_{\alpha \sigma} \int d \omega f(\omega) \operatorname{Im}\left[G_{d \alpha \sigma}(\omega) \omega \frac{\partial \Delta_{\alpha}(\omega)}{\partial \omega}\right],
\end{aligned}
$$

and $G_{d \alpha \sigma}(\omega)$ is the retarded Green function for local level $\alpha$. Combining $E_{\text {int }}^{(1)}$ with $E_{\text {hyb }}$ gives for the impurity internal energy

$$
E_{\mathrm{imp}}=E_{\mathrm{occ}}+E_{\mathrm{C}}+\frac{1}{2} E_{\mathrm{hyb}}+E_{\mathrm{int}}^{(2)},
$$

where, as before, all contributions except the last one are evaluated as local static correlation functions. For reasons discussed in Sec. III, the temperature dependence of the last term is negligible in many cases and the impurity specific heat can be calculated to high accuracy via

$$
\begin{aligned}
C_{\mathrm{imp}} & =\frac{\partial E_{\mathrm{occ}}}{\partial T}+\frac{\partial E_{\mathrm{C}}}{\partial T}+\frac{1}{2} \frac{\partial E_{\mathrm{hyb}}}{\partial T} \\
& =\frac{\partial E_{\text {ionic }}}{\partial T}+\frac{1}{2} \frac{\partial E_{\mathrm{hyb}}}{\partial T},
\end{aligned}
$$

where $E_{\text {ionic }}=\left\langle H_{\text {imp }}\right\rangle$.

\section{B. Dissipative two-state systems and the anisotropic Kondo model}

The method of Sec. III can be applied to bosonic models such as the dissipative two-state system, ${ }^{4,5}$ and for Ohmic dissipation, one can further relate the results to the AKM and related models (e.g., a two-level system in a metallic environment ${ }^{59}$ ). Dissipative two-state systems are of interest in many contexts, including the description of qubits coupled to their environment.

The Hamiltonian of the dissipative two-state system is given by $H=H_{\mathrm{S}}+H_{\mathrm{B}}+H_{\mathrm{I}}$. The first term $H_{\mathrm{S}}=-\frac{1}{2} \Delta_{0} \sigma_{x}+$ $\frac{1}{2} \epsilon \sigma_{z}$ describes a two-level system with bias splitting $\epsilon$ and tunneling amplitude $\Delta_{0}$, and $\sigma_{i=x, y, z}$ are Pauli spin matrices. $H_{\mathrm{B}}=\sum_{i} \omega_{i}\left(a_{i}^{\dagger} a_{i}+1 / 2\right)$ is the environment and consists of an infinite set of harmonic oscillators $(i=$ $1,2, \ldots, \infty)$ with $a_{i}\left(a_{i}^{\dagger}\right)$ the annihilation (creation) operators for a harmonic oscillator of frequency $\omega_{i}$ and $0 \leqslant \omega_{i} \leqslant \omega_{c}$, where $\omega_{c}$ is an upper cut-off frequency. The noninteracting density of states of the environment is denoted by $g\left(\omega_{i}\right)=\sum_{i} \delta\left(\omega-\omega_{i}\right)$ and is finite in the interval $\left[0, \omega_{\mathrm{c}}\right]$ and zero otherwise. Finally, $H_{\mathrm{I}}=\frac{1}{2} \sigma_{z} \sum_{i} \lambda_{i}\left(a_{i}+a_{i}^{\dagger}\right)$ describes the coupling of the two-state system coordinate $\sigma_{z}$ to the oscillators, with $\lambda_{i}$ denoting the coupling strength to oscillator $i$. The function $\Gamma(\omega+i \delta)=\sum_{i}\left(\lambda_{i} / 2\right)^{2} /\left(\omega-\omega_{i}+\right.$ $i \delta)=\int d \omega^{\prime}\left(\lambda\left(\omega^{\prime}\right) / 2\right)^{2} g\left(\omega^{\prime}\right) /\left(\omega-\omega^{\prime}+i \delta\right)$ characterizes the system-environment interaction. The Ohmic two-state system, specified by a spectral function $J(\omega)=-\frac{1}{\pi} \operatorname{Im} \Gamma(\omega+i \delta) \sim$ $\alpha \omega$ for $\omega \rightarrow 0$, where $\alpha$ is the dimensionless dissipation strength, is equivalent to the AKM $H=\sum_{k \sigma} \epsilon_{k} c_{k \sigma}^{\dagger} c_{k \sigma}+$ $\frac{J_{\perp}}{2}\left(S^{+} s_{0}^{-}+S^{-} s_{0}^{+}\right)+J_{\|} S_{z} s_{0}^{z}+B S_{z}$, where $J_{\perp}\left(J_{\|}\right)$is the transverse (longitudinal) part of the Kondo exchange interaction and $B$ is a local magnetic field. The correspondence is given by $\rho J_{\perp}=-\Delta_{0} / \omega_{\mathrm{c}}$ and $\alpha=(1+2 \delta / \pi)^{2}$, where $\delta=\arctan \left(-\pi \rho J_{\|} / 4\right)$ and $\rho$ is the density of states of the conduction electrons in the AKM. ${ }^{4,5,60-62}$ The low-energy scale of the Ohmic two-state system is the renormalized tunneling amplitude $\Delta_{\mathrm{r}}$ given by $\Delta_{\mathrm{r}} / \omega_{\mathrm{c}}=\left(\Delta_{0} / \omega_{\mathrm{c}}\right)^{1 /(1-\alpha)}$ and corresponds to the low-energy Kondo scale $T_{\mathrm{K}}$ of the AKM. Special care is needed to obtain results for the Ohmic two-state system from the AKM in the vicinity of the singular point $\alpha \rightarrow 1^{-}$since this corresponds to $J_{\|} \rightarrow 0$ but with the condition $0<J_{\perp}<J_{\|}$, that is, in terms of parameters of the Ohmic two-state system one requires $\Delta_{0} / \omega_{\mathrm{c}} \ll 1-\alpha \ll 1$ in order to investigate the vicinity of $\alpha=1$ within the AKM. ${ }^{5}$

The specific heat $C_{\text {imp }}=\partial E_{\text {imp }} / \partial T$ of the Ohmic two-state system is defined via an impurity internal energy $E_{\text {imp }}=$ $E_{\text {total }}-E_{0}$, where $E_{\text {total }}=\langle H\rangle=\left\langle H_{\mathrm{S}}\right\rangle+\left\langle H_{\mathrm{B}}\right\rangle+\left\langle H_{\mathrm{I}}\right\rangle$ and $E_{0}=\left\langle H_{\mathrm{B}}\right\rangle_{0}=\sum_{i} \omega_{i}\left\langle a_{i}^{\dagger} a_{i}\right\rangle_{0}+E_{\mathrm{zp}}=\int_{0}^{\omega_{c}} d \omega \omega n(\omega) g(\omega)+$ $E_{\mathrm{zp}}$, where $n(\omega)=1 /\left(e^{\beta \omega}-1\right)$ is the Bose distribution function and the zero point energy $E_{\mathrm{zp}}$ can be dropped, as it cancels in the difference $\left\langle H_{\mathrm{B}}\right\rangle-E_{0}=E_{\mathrm{B}}-E_{0}$ appearing in $E_{\text {imp. }}$. Evaluating $E_{\mathrm{B}}-E_{0}$ and $E_{\mathrm{I}}=\left\langle H_{\mathrm{I}}\right\rangle$ following the approach in Sec. III we find

$$
\begin{aligned}
E_{\mathrm{B}}-E_{0} & =\frac{1}{\pi} \int d \omega n(\omega) \operatorname{Im}\left\{\chi_{z z}(\omega+i \delta) \frac{\partial}{\partial \omega}[\omega \Gamma(\omega+i \delta)]\right\} \\
& =E_{\mathrm{B}}^{(1)}+E_{\mathrm{B}}^{(2)}, \\
E_{\mathrm{B}}^{(1)} & =\frac{1}{\pi} \int d \omega n(\omega) \operatorname{Im}\left[\chi_{z z}(\omega+i \delta) \Gamma(\omega+i \delta)\right], \\
E_{\mathrm{B}}^{(2)} & =\frac{1}{\pi} \int d \omega n(\omega) \operatorname{Im}\left[\chi_{z z}(\omega+i \delta) \omega \frac{\partial \Gamma(\omega+i \delta)}{\partial \omega}\right],
\end{aligned}
$$


and

$$
E_{\mathrm{I}}=-\frac{1}{\pi} \int d \omega n(\omega) \operatorname{Im}\left[\chi_{z z}(\omega+i \delta) \Gamma(\omega+i \delta)\right],
$$

where $\chi_{z z}(\omega+i \delta)=\left\langle\left\langle\sigma_{z} ; \sigma_{z}\right\rangle\right\rangle_{\omega+i \delta}$ is the longitudinal retarded dynamic susceptibility and $\Gamma(\omega+i \delta)$, characterizing the system-environment interaction, was defined above. Noting that $E_{\mathrm{B}}^{(1)}$ exactly cancels $E_{\mathrm{I}}$ in the impurity internal energy, we find

$$
E_{\mathrm{imp}}=-\frac{1}{2} \Delta_{0}\left\langle\sigma_{x}\right\rangle+\frac{1}{2} \epsilon\left\langle\sigma_{z}\right\rangle+E_{\mathrm{B}}^{(2)},
$$

that is, $E_{\mathrm{imp}}=E_{\mathrm{S}}+E_{\mathrm{B}}^{(2)}$. The term $E_{\mathrm{B}}^{(2)}$ gives a non-negligible contribution to the impurity internal energy. For example, in the Ohmic case with spectral function $J(\omega)=-\frac{1}{\pi} \operatorname{Im} \Gamma(\omega+$ $i \delta) \sim \alpha \omega$ we have $\omega \partial J(\omega) / \partial \omega \sim \alpha \omega$ at low frequencies, so $E_{\mathrm{B}}^{(2)}$ provides a contribution proportional to $\alpha$. By carrying out specific heat calculations on the AKM, we find numerically that the impurity specific heat is consistent with setting $E_{\mathrm{B}}^{(2)}=$ $\frac{1}{2} \alpha \Delta_{0}\left\langle\sigma_{x}\right\rangle+A$, with $A$ being a weakly temperature-dependent term, and negligible for calculating the specific heat, except in the limit $\alpha \rightarrow 1^{-}$. The latter limit is difficult to treat numerically because of the vanishing low-energy scale $\Delta_{\mathrm{r}}$ for $\alpha \rightarrow 1^{-}$(e.g., for $\Delta_{0} / \omega_{\mathrm{c}}=0.01$ and $\alpha=0.9$ we have $\left.\Delta_{\mathrm{r}} / \omega_{\mathrm{c}}=10^{-20}\right)$. Hence, except in this extreme limit, and as we show below by comparing with exact results, the impurity specific heat can be obtained accurately from $C_{\text {imp }}=\frac{\partial E_{\text {imp }}}{\partial T}$ by using

$$
E_{\mathrm{imp}} \approx-\frac{1}{2} \Delta_{0}(1-\alpha)\left\langle\sigma_{x}\right\rangle+\frac{1}{2} \epsilon\left\langle\sigma_{z}\right\rangle .
$$

Figure 12 shows results obtained in this way for $C_{\text {imp }}(T) /\left(k_{\mathrm{B}} T / \Delta_{\mathrm{r}}\right)$ compared to Bethe ansatz calculations for the $\mathrm{AKM}^{63}$ for a range of dissipation strengths. These

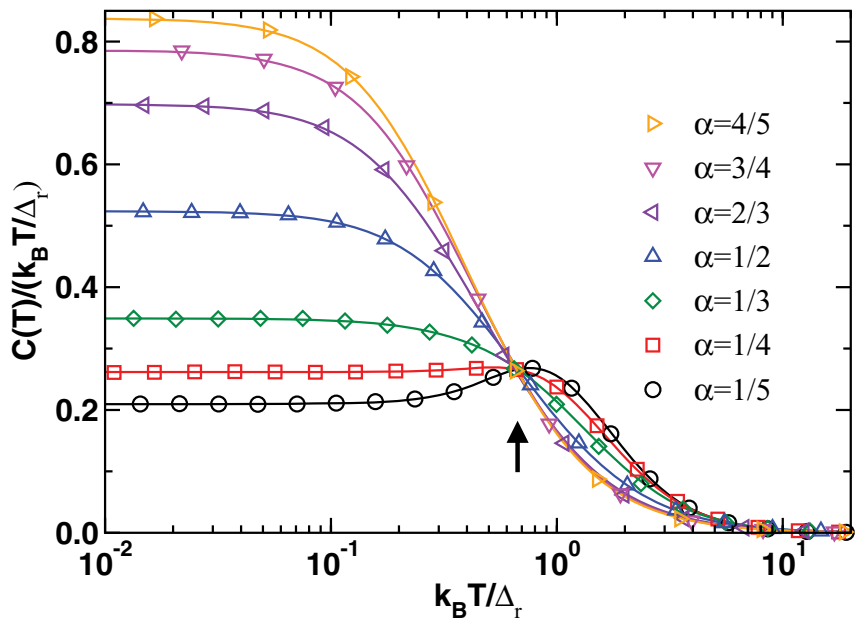

FIG. 12. (Color online) Specific heat $C_{\mathrm{imp}}(T) / k_{\mathrm{B}} T / \Delta_{\mathrm{r}}$ of the Ohmic two-state system as a function of reduced temperature $k_{\mathrm{B}} T / \Delta_{\mathrm{r}}$ for a range of dissipation strengths $\alpha=1 / 5,1 / 4$, $1 / 3,1 / 2,2 / 3,3 / 4,4 / 5$. Symbols: NRG results in new approach. Lines: Bethe ansatz results. The renormalized tunneling amplitude $\Delta_{\mathrm{r}}$ from the Bethe ansatz is used. The vertical arrow indicates the approximate crossing point at $k_{\mathrm{B}} T / \Delta_{\mathrm{r}} \approx 0.67$. Model parameters: $\Delta_{0} / \omega_{\mathrm{c}}=0.005$. NRG parameters: $\Lambda=10, n_{z}=4$ retaining 860 states per NRG iteration.

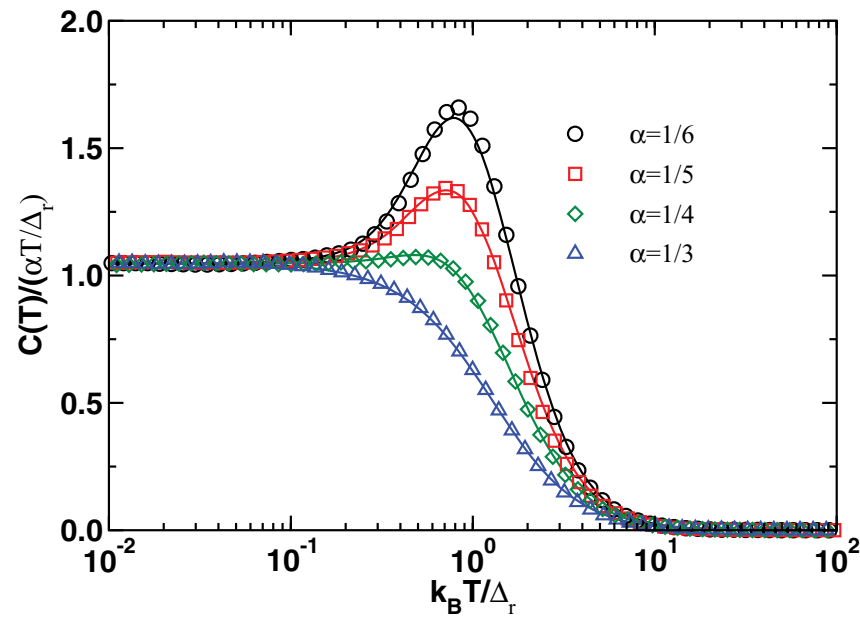

FIG. 13. (Color online) Specific heat $C_{\text {imp }}(T) /\left(\alpha k_{\mathrm{B}} T / \Delta_{\mathrm{r}}\right)$ of the Ohmic two-state system as a function of reduced temperature $k_{\mathrm{B}} T / \Delta_{\mathrm{r}}$ for a range of dissipation strengths $\alpha<1 / 2$. Symbols: NRG results in new approach. Lines: Bethe ansatz results. In the low-temperature Fermi liquid regime $T \ll \Delta_{\mathrm{r}}$ we have $C_{\text {imp }}(T) /\left(\alpha k_{\mathrm{B}} T / \Delta_{\mathrm{r}}\right)=\tilde{\gamma}+$ $\tilde{\beta}\left(T / \Delta_{\mathrm{r}}\right)^{2}$, with $\tilde{\gamma}=\pi / 3$ and the $T^{3}$ coefficient in $C(T)$ changes sign for $\alpha<1 / 3$ (see Ref. 62). Model parameters: $\Delta_{0} / \omega_{\mathrm{c}}=0.005$. NRG parameters: $\Lambda=10, n_{z}=4$ retaining 860 states per NRG iteration.

results recover the known results for asymptotically high and low temperatures. ${ }^{64}$ In common with specific heats of other correlated electron systems as a function of interaction strength, ${ }^{65}$ we observe a crossing point in $C(T) / T$ (here, at $\left.k_{\mathrm{B}} T / \Delta_{\mathrm{r}} \approx 0.67\right)$. On decreasing the dissipation strength from strong $(\alpha>1 / 2)$ to weak values $(\alpha<1 / 2)$ the $T^{3}$ coefficient of the specific heat changes sign for $\alpha<1 / 3$ resulting in the appearance of a finite temperature peak in $C(T) / T$. This is shown in more detail in Fig. 13. It signifies the development of a gap $\sim \Delta_{0}$ in the spectrum as $\alpha \rightarrow 0$. For $\alpha=0$ one eventually recovers the Schottky specific heat for a noninteracting two-level system. The expression (31) for the Ohmic two system is also the impurity internal energy of the equivalent AKM (indeed, the NRG results that we showed were for this model). The correspondence of model parameters was given above and the operators $\sigma_{x}$ and $\sigma_{z}$ are identified, under bosonization, ${ }^{4,5,60,62}$ with the spin-flip operator $S^{+} s_{0}^{-}+S^{-} s_{0}^{+}$and the local $S_{z}$ in the AKM, respectively. The zero temperature expectation values $\left\langle\sigma_{x}\right\rangle$ and $\left\langle\sigma_{z}\right\rangle$ (and the associated entanglement entropy of the qubit) have been studied previously as a function of dissipation strength and finite bias. ${ }^{66,67}$

We expect that the term $E_{\mathrm{B}}^{(2)}$ is non-negligible also for generic spectral functions $J(\omega) \sim \omega^{s}$ and certainly for the subOhmic case $s<1$. Recent results for the local spin dynamics of the sub-Ohmic spin boson model ${ }^{68}$ could shed light on this.

The result (31) shows that a significant contribution to the impurity internal energy and specific heat arises from the (interacting) bath contribution $E_{\mathrm{B}}^{(2)}$, which remains finite for arbitrarily small $\alpha$. Thus, while a definition of the internal energy of the system via $E_{\mathrm{S}}=\left\langle H_{\mathrm{S}}\right\rangle$ and the specific heat via $C_{\mathrm{S}}=\partial E_{\mathrm{S}} / \partial T$, might seem reasonable for a small quantum 
system weakly coupled to an infinite bath, such a definition yields, in general, a specific heat $C_{\mathrm{S}}$ which differs from $C_{\text {imp }}{ }^{69-72}$ One system for which the two definitions agree is the harmonic oscillator coupled Ohmically to an infinite bath of harmonic oscillators. ${ }^{69}$ This result, however, represents a special case, and, moreover, is sensitive to details of the cut-off scheme used for the spectral function $J(\omega)$ (see Refs. 69 and 72). The use of $E_{\mathrm{imp}}$ and $C_{\mathrm{imp}}$ as definitions for the system internal energy and specific heat in the context of open quantum systems ${ }^{5,73}$ also provides an unambiguous prescription for their measurement in terms of two separate measurements, ${ }^{71,74}$ one for $H$ and one for $H_{0}$. We note also that the impurity specific heat $C_{\text {imp }}(T)=C(T)-C_{0}(T)$ need not be positive at all temperatures and only the positivity of $C(T)$ and $C_{0}(T)$ in Eqs. (1) and (2) is guaranteed by thermodynamic stability of the equilibrium systems described by $H$ and $H_{0}$ (see Ref. 75). Examples of systems where the difference $C_{\text {imp }}(T)$ may be negative in some temperature range, include quantum impurities exhibiting a flow between a stable and an unstable fixed point, ${ }^{76}$ and magnetic impurities in superconductors. ${ }^{77}$

\section{DISCUSSION AND CONCLUSIONS}

In this paper we introduced a new approach to the calculation of impurity internal energies and specific heats of quantum impurity models within the NRG method. For general Anderson impurity models, the impurity contribution to the internal energy was expressed in terms of local quantities and the main contribution to the impurity specific heat was shown to arise from local static correlation functions. For this class of models, the impurity specific heat can be obtained essentially exactly as $C_{\text {imp }}(T)=\frac{\partial E_{\text {ionic }}}{\partial T}+\frac{1}{2} \frac{\partial E_{\text {hyb }}}{\partial T}$, where $E_{\text {ionic }}=\left\langle H_{\text {imp }}\right\rangle$ and $E_{\text {hyb }}$ is the hybridization energy. A comparison with exact Bethe ansatz calculations showed that the results for specific heats of the Anderson impurity model are recovered accurately over the whole temperature and magnetic field range. The new method has several advantages over the conventional approach to specific heats within the NRG, namely, (i) only diagonalization of the total system is required, (ii) only local quantities are required, and (iii) discretization oscillations at large $\Lambda$ are significantly smaller than in the conventional approach.

For the dissipative two-state system we obtain the specific heat as $C_{\mathrm{imp}}(T) \equiv \frac{\partial E_{\mathrm{imp}}}{\partial T}=\frac{\partial E_{\mathrm{S}}}{\partial T}+\frac{\partial E_{\mathrm{B}}^{(2)}}{\partial T}$, where $E_{\mathrm{S}}=\left\langle H_{\mathrm{S}}\right\rangle$ is analogous to $E_{\text {ionic }}$ in the Anderson model, and $E_{\mathrm{B}}^{(2)}$ is a contribution to the energy of the system arising from the interaction with the bath. It depends on the local dynamical susceptibility and the type of coupling to the environment. For the Ohmic case we used the equivalence of the Ohmic two-state system to the AKM to show numerically that $E_{\mathrm{B}}^{(2)}=$ $\frac{1}{2} \alpha \Delta_{0}\left\langle\sigma_{x}\right\rangle+A$, with $A$ having a negligible temperature dependence, except in the extreme limit $\alpha \rightarrow 1^{-}$. Comparison with exact Bethe ansatz calculations on the AKM confirmed the above.

The approach described in this paper applies to energydependent hybridizations also, see Fig. 7, so inclusion of the term $E_{\text {int }}^{(2)}$ in Eq. (14) could prove useful in applications to quantum impurities with a pseudogap density of states. ${ }^{37,78}$
It may also be applied within other methods for solving quantum impurity models, for example, within continuous time $^{79}$ or Hirsch-Fey ${ }^{80}$ quantum Monte Carlo techniques or exact diagonalization methods (for a recent review see Ref. 81 and references therein). Local static correlation functions, such as the double occupancy, required for $E_{\text {imp }}$, are readily extracted within these approaches. ${ }^{82}$

Within a DMFT treatment of correlated lattice models, ${ }^{19-22}$ the hybridization function $\Delta$ acquires an important temperature and frequency dependence $\Delta(\omega) \rightarrow \Delta(\omega, T)$. The latter enters explicitly in the term $E_{\text {int }}^{(2)}$, whose inclusion could offer an approach to the calculation of specific heats of correlated lattice models. The thermodynamic potential of the latter $^{83}$ is a sum of two parts, one depending on the local self-energy, which is the central quantity calculated in DMFT, and another equal to the thermodynamic potential $\Omega_{\text {imp }}=$ $E_{\text {imp }}-T S_{\text {imp }}$ of the effective impurity model. The latter can be obtained from $E_{\text {imp }}(T)$, via $C_{\text {imp }}(T)$ and $S_{\text {imp }}(T)=$ $\int_{0}^{T} d T^{\prime} \frac{C_{\text {imp }}\left(T^{\prime}\right)}{T^{\prime}}$. The impurity internal energy, expressed in terms of local dynamical quantities as in Ref. 45, has recently been used in a DMFT solution of the Hubbard model within a variational generalization ${ }^{84}$ of the local moment approach. $^{85}$

In the future it may be interesting, especially in the context of qubits or nanodevices, to consider the time dependence of the impurity internal energy subject to an initial state preparation, for example, within techniques such as timedependent density matrix renormalization group ${ }^{86-88}$ or timedependent NRG. ${ }^{33,89,90}$

\section{ACKNOWLEDGMENTS}

We thank D. P. DiVincenzo, A. Rosch, S. Kirchner, A. Weichselbaum, G.-L. Ingold, P. Hänggi, and A. Liebsch for useful discussions and comments on this work, and A. Kauch for drawing our attention to Ref. 45. We acknowledge supercomputer support by the John von Neumann Institute for Computing (Jülich).

\section{APPENDIX A: BAND CONTRIBUTION TO IMPURITY INTERNAL ENERGY}

The expression (11) for the conduction band contribution to the impurity internal energy requires evaluation of the integral

$$
I(\omega)=\int d \epsilon \frac{\epsilon V^{2} N(\epsilon)}{(\omega-\epsilon+i \delta)^{2}} .
$$

We assume a density of states $N(\omega)$ vanishing at the band edges at $\omega= \pm D$. The hybridization function $\Delta(\omega)=$ $\sum_{k} V^{2} /\left(\omega-\epsilon_{k}+i \delta\right)=\Delta_{R}(\omega)+i \Delta_{I}(\omega)$, where $\Delta_{I}(\omega)=$ $-\pi N(\omega) V^{2}$. With these definitions, we have

$$
\begin{aligned}
I(\omega)= & -\frac{1}{\pi} \int_{-D}^{+D} d \epsilon \epsilon \Delta_{I}(\epsilon) \frac{\partial}{\partial \epsilon} \frac{1}{(\omega-\epsilon+i \delta)} \\
= & -\left.\frac{1}{\pi} \frac{\epsilon \Delta_{I}(\epsilon)}{\omega-\epsilon+i \delta}\right|_{-D} ^{+D} \\
& +\frac{1}{\pi} \int_{-D}^{+D} d \epsilon \frac{1}{\omega-\epsilon+i \delta} \frac{\partial}{\partial \epsilon}\left[\epsilon \Delta_{I}(\epsilon)\right] .
\end{aligned}
$$


The first term vanishes since $\Delta_{I}( \pm D)=0$ for regular (e.g., $3 D$ ) densities of states (and will otherwise result in contributions with negligible temperature dependence). The second term can be evaluated by noting that $\Delta(\omega+i \delta)$ satisfies the causal properties of retarded Green functions and by using the following properties of principle value (P.V.) integrals: If P.V. $[f(x)]=g(y)$ then P.V. $\left[f^{\prime}(x)\right]=g^{\prime}(y)$ and P.V. $[x f(x)]=$ $y g(y)+\frac{1}{\pi} \int d x f(x)$. The final result is

$$
I(\omega)=-\frac{\partial}{\partial \omega}[\omega \Delta(\omega)] .
$$

\section{APPENDIX B: NUMERICAL SOLUTION OF THE THERMODYNAMIC BETHE ANSATZ EQUATIONS}

In this Appendix we summarize the thermodynamic Bethe ansatz (TBA) equations for the Anderson model, which were derived by Okiji and Kawakami ${ }^{42,91,92}$ and Tsvelick, Filyov, and Wiegmann, ${ }^{40,41,93,94}$ and provide details of their numerical solution. ${ }^{46-49,62,95}$ The numerical procedure described applies to both the symmetric and asymmetric Anderson models and in the presence of a finite magnetic field and was used to obtain the results presented in this paper.

\section{Thermodynamic Bethe ansatz equations}

The thermodynamic Bethe ansatz (TBA) produces an infinite set of coupled integral equations for the functions $\epsilon(k)$, $\kappa_{n}^{\prime}(\Lambda)$, and $\kappa_{n}(\Lambda), n=1,2, \ldots$, describing the charge and spin excitations of the system (Tsvelick and Wiegmann ${ }^{40}$ ):

$$
\begin{aligned}
& \epsilon(k)-T \int_{-\infty}^{\infty} s[g(k)-\Lambda] \ln \left[f\left(\kappa_{1}(\Lambda)\right] d \Lambda\right. \\
& =\epsilon_{0}(k)-T \int_{-\infty}^{\infty} s[g(k)-\Lambda] \ln \left[f\left(\kappa_{1}^{\prime}(\Lambda)\right] d \Lambda,\right. \\
& \kappa_{n}(\Lambda)+T\left(s *\left\{\ln \left[f\left(\kappa_{n+1}\right)\right]+\ln \left[f\left(\kappa_{n-1}\right)\right]\right\}\right)(\Lambda) \\
& =\delta_{n, 1} T \int_{-\infty}^{\infty} s[g(k)-\Lambda] \ln \{f[-\epsilon(k)]\} g^{\prime}(k) d k, \\
& \kappa_{n}^{\prime}(\Lambda)+T\left(s *\left\{\ln \left[f\left(\kappa_{n+1}^{\prime}\right)\right]+\ln \left[f\left(\kappa_{n-1}^{\prime}\right)\right]\right\}\right)(\Lambda) \\
& =\delta_{n, 1} T \int_{-\infty}^{\infty} s[g(k)-\Lambda] \ln \{f[\epsilon(k)]\} g^{\prime}(k) d k,
\end{aligned}
$$

where

$$
\begin{aligned}
g(k) & =\frac{\left(k-\varepsilon_{d}-\frac{1}{2} U\right)^{2}}{2 \Gamma \mathrm{U}}, \quad s(\Lambda)=\frac{1}{2 \cosh (\pi \Lambda)}, \\
f(k) & =\frac{1}{1+\mathrm{e}^{k / T}}, \quad R(x)=\frac{1}{\pi} \int_{0}^{\infty} \frac{\cos (\omega x)}{1+\mathrm{e}^{\omega}} d \omega \\
\epsilon_{0}(k) & =k-\varepsilon_{d}-\frac{1}{2} U+\int_{-\infty}^{\infty} R[g(k)-g(p)] p \cdot g^{\prime}(p) d p
\end{aligned}
$$

$g^{\prime}(k)$ denotes the first derivative of $g(k)$ with respect to $k$. * is the convolution of two functions. $\kappa_{0}$ and $\kappa_{0}^{\prime}$ equal $-\infty$. For $n \rightarrow \infty$ the functions approach the constant values,

$$
\lim _{n \rightarrow \infty} \kappa_{n}=n \cdot H, \quad \lim _{n \rightarrow \infty} \kappa_{n}^{\prime}=n \cdot\left(2 \varepsilon_{d}+U\right),
$$

where $H$ is a uniform magnetic field and $2 \varepsilon_{d}+U$ measures the deviation from the symmetric point at $\varepsilon_{d}=-U / 2$. The impurity contribution to the specific heat $C_{\mathrm{imp}}$ may be calculated from the the impurity contribution to the thermodynamic potential $\Omega_{\text {imp }}$ via $C_{\text {imp }}=-T \partial^{2} \Omega_{\text {imp }} / \partial T^{2}$, where

$$
\begin{aligned}
\Omega_{\mathrm{imp}}= & T \int_{-\infty}^{\infty} \rho_{0}(k) \ln \{f[-\epsilon(k)]\} d k \\
& +T \int_{-\infty}^{\infty} \sigma_{0}(\Lambda) \ln \left[\kappa_{1}^{\prime}(\Lambda)\right] d \Lambda+E_{0} .
\end{aligned}
$$

The functions $\rho_{0}$ and $\sigma_{0}$ are given by

$$
\begin{aligned}
\sigma_{0}(\Lambda) & =\int_{-\infty}^{\infty} s[\Lambda-g(k)] \Delta(k) d k, \\
\rho_{0}(k) & =\Delta(k)+g^{\prime}(k) \int_{-\infty}^{\infty} R[g(k)-g(p)] \Delta(p) d p,
\end{aligned}
$$

where $\Delta(k)=\frac{\Gamma}{\pi\left[\Gamma^{2}+\left(k-\varepsilon_{d}\right)^{2}\right]} . E_{0}$ is the ground-state energy of the symmetric Anderson model. ${ }^{91}$ Note two changes with respect to the earlier Ref. 40: A sign change in Eq. (B1c) (as in Wiegmann and Tsvelick ${ }^{41}$ ) and a factor 2 in the boundary value for $\kappa_{n}^{\prime}$ in Eq. (B2) (as in Okiji and Kawakami ${ }^{42}$ ).

For the calculations we use a transformation of $\kappa_{n}$ and $\kappa_{n}^{\prime}$ to new functions $\xi_{n}$ and $\xi_{n}^{\prime}$ similar to that used in previous works. ${ }^{46,47,62}$ After substituting $\xi_{n}=\ln \left(1+\mathrm{e}^{\kappa_{n} / T}\right)$ and $\xi_{n}^{\prime}=$ $\ln \left(1+\mathrm{e}^{\kappa_{n}^{\prime} / T}\right)$ we obtain the following coupled equations:

$$
\begin{aligned}
& \xi_{1}(\Lambda)=\ln \left(1+\exp \left\{\left[s *\left(\xi_{2}+I_{1}\right)\right](\Lambda)\right\}\right), \\
& \xi_{n}(\Lambda)=\ln \left(1+\exp \left\{\left[s *\left(\xi_{n-1}+\xi_{n+1}\right)\right](\Lambda)\right\}\right), \\
& \xi_{1}^{\prime}(\Lambda)=\ln \left(1+\exp \left\{\left[s *\left(\xi_{2}^{\prime}+I_{1}^{\prime}\right)\right](\Lambda)\right\}\right), \\
& \xi_{n}^{\prime}(\Lambda)=\ln \left(1+\exp \left\{\left[s *\left(\xi_{n-1}^{\prime}+\xi_{n+1}^{\prime}\right)\right](\Lambda)\right\}\right), \\
& I_{1}(\Lambda)=\int_{-\infty}^{\infty} s[g(k)-\Lambda] \ln \{f[-\epsilon(k)]\} g^{\prime}(k) d k, \\
& I_{1}^{\prime}(\Lambda)=\int_{-\infty}^{\infty} s[g(k)-\Lambda] \ln \{f[\epsilon(k)]\} g^{\prime}(k) d k, \\
& I(k)=\int_{-\infty}^{\infty} s[g(k)-\Lambda]\left[\xi_{1}(\Lambda)-\xi_{1}^{\prime}(\Lambda)\right] d \Lambda, \\
& e(k)=e_{0}(k)+T \cdot I(k) .
\end{aligned}
$$

\section{Truncation}

For calculational purposes the equations $\xi_{n}$ and $\xi_{n^{\prime}}^{\prime}$ have to be truncated at some finite value $n=N$ and $n^{\prime}=N^{\prime}$. One has to calculate the functions at the truncation with care, to avoid wrong results at the boundaries $\Lambda \rightarrow \pm \infty$. We use the truncation scheme of Takahashi and Shiroishi. ${ }^{95}$ It is assumed that the function $s(x)$ can be approximated by $\delta(x) / 2$ for large $n$ or $n^{\prime}$. This is justified as the functions become smoother in this region (see Fig. 14). Rewritten for the Anderson model and for $\xi_{N}$ and $\xi_{N}^{\prime}$ the corresponding truncation functions are 


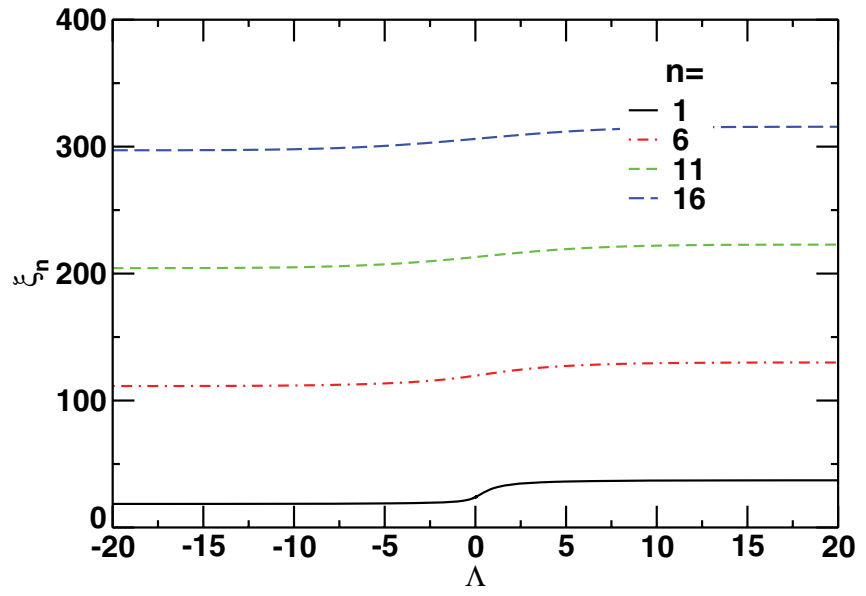

FIG. 14. (Color online) The figure shows a set of $\xi_{n}$ for the symmetric case $\left(\varepsilon_{d}+U / 2=0\right)$ zoomed to range of $\Lambda=-20, \ldots, 20$. The functions become smoother with higher $n$ due to the convolution with $s(x)$.

calculated by

$$
\begin{aligned}
& \xi_{N}= \ln \left\{\left[\cosh \left(\frac{H}{2}\right) \sqrt{2+\mathrm{e}^{\xi_{N-1}}}\right.\right. \\
&+ \sqrt{\left.\left.1+\sinh ^{2}\left(\frac{H}{2}\right)\left(2+e^{\xi_{N-1}}\right)\right]^{2}\right\},} \\
& \xi_{N^{\prime}}^{\prime}=\ln \left\{\left[\cosh \left(\frac{2 \varepsilon_{d}+U}{2}\right) \sqrt{2+e^{\xi_{N^{\prime}-1}^{\prime}}}\right.\right. \\
&\left.\left.+\sqrt{1+\sinh ^{2}\left(\frac{2 \varepsilon_{d}+U}{2}\right)\left(2+e^{\xi_{N^{\prime}-1}}\right)}\right]^{2}\right\} .
\end{aligned}
$$

As a further check, and to ensure the correct behavior at the boundaries, the TBA integral equations were explicitly solved in the limits of $\Lambda, k \rightarrow \pm \infty$. As the functions are smooth in this limit one can assume that $s(x) \rightarrow \delta(x) / 2$ and $\lim _{k \rightarrow \infty} \epsilon_{0}(k)=2\left(k-\varepsilon_{d}-U / 2\right), \lim _{k \rightarrow-\infty} \epsilon_{0}(k)=0$. This leads to the following set of coupled algebraic equations:

$$
\begin{aligned}
\lim _{\Lambda \rightarrow-\infty} & \\
\xi_{1} & =\ln \left[1+\exp \left(\frac{1}{2} \xi_{2}\right)\right], \\
\xi_{n} & =\ln \left\{1+\exp \left[\frac{1}{2}\left(\xi_{n-1}+\xi_{n+1}\right)\right]\right\}, \\
\xi_{1}^{\prime} & =\ln \left[1+\exp \left(\frac{1}{2} \xi_{2}^{\prime}\right)\right], \\
\xi_{n}^{\prime} & =\ln \left\{1+\exp \left[\frac{1}{2}\left(\xi_{n-1}^{\prime}+\xi_{n+1}^{\prime}\right)\right]\right\},
\end{aligned}
$$

$$
\begin{aligned}
\lim _{\Lambda \rightarrow \infty} & \\
\xi_{1} & =\ln \left[1+\exp \left(\frac{1}{2}\left\{\xi_{2}-\ln \left[1+\exp \left(\frac{1}{2} \xi_{1}\right)\right]\right\}\right)\right], \\
\xi_{n} & =\ln \left\{1+\exp \left[\frac{1}{2}\left(\xi_{n-1}+\xi_{n+1}\right)\right]\right\}, \\
\xi_{1}^{\prime} & =0, \\
\xi_{n}^{\prime} & =\ln \left\{1+\exp \left[\frac{1}{2}\left(\xi_{n-1}^{\prime}+\xi_{n+1}^{\prime}\right)\right]\right\} .
\end{aligned}
$$

The truncation constants $\xi_{N}$ and $\xi_{N^{\prime}}^{\prime}$ are calculated as in Eq. (B5). The boundary values were calculated by iteration using a modification of the Powell hybrid method.

\section{Numerical details}

For the calculations, a logarithmic grid was used that is centered around $\varepsilon_{d}+U / 2$. The TBA equations were solved by iteration. The initial values of $\xi_{n}$ and $\xi_{n^{\prime}}^{\prime}$ were chosen to fit a tanh function with boundary values given by the correct boundary values of $\xi_{n}$ and $\xi_{n}^{\prime}$, obtained as described above. The integrations were carried out using adaptive routines with the integrands being represented by splines of smooth functions only (see below). A smoother convergence of the iteration procedure is obtained by using $10 \%$ of the old iteration values in each step. To represent only smooth functions as splines, $\xi_{1}$ and $\xi_{1}^{\prime}$ are not interpolated, but instead the $s * \xi_{2}$ and $s * \xi_{2}^{\prime}$, respectively. The values of $\xi_{1}$ and $\xi_{1}^{\prime}$ are then calculated from these convolutions and from $I_{1}$ and $I_{1}^{\prime}$ using Eqs. (B4a) and (B4c). This avoids numerical problems due to the exponential drop to zero of $\xi_{1}^{\prime}$ beyond a certain rapidity $\Lambda_{0}$. See Fig. 15 for a comparison between the behavior of $\xi_{1}^{\prime}$ and $I_{1}^{\prime} . N=N^{\prime}=20$ functions were used and iterated 500 times for the figures in this Appendix (and 2000 times for results in the paper). The growth rate of the grid was 1.05 and it consisted of 801 points. The mid 400 values lie in a range of $[-40,40]$. After a certain temperature-dependent cutoff $\left( \pm 40 \pm 40 \cdot T / T_{0}\right)$ the boundary values were used instead of being calculated to ensure numerical stability. The thermodynamic potential was calculated in a range of $T_{0} \times 10^{-3}$ to $T_{0} \times 10^{6}$ on a logarithmic mesh (factor $2^{1 / 8}$ as step width) where $T_{0}$ is defined as $T_{0}=\sqrt{U \Gamma / 2} \exp \left(-\frac{\pi U}{8 \Gamma}+\frac{\pi \Gamma}{2 U}\right)$, Kondo temperature for the symmetric case. It is related to the magnetic susceptibility at zero temperature $\chi_{\mathrm{imp}}(T=0)=\frac{\left(g \mu_{\mathrm{B}}\right)^{2}}{4 k_{\mathrm{B}} T_{0}}$ (see Hewson in Ref. 1 p. 165, and Kawakami and Okiji in Ref. 96).

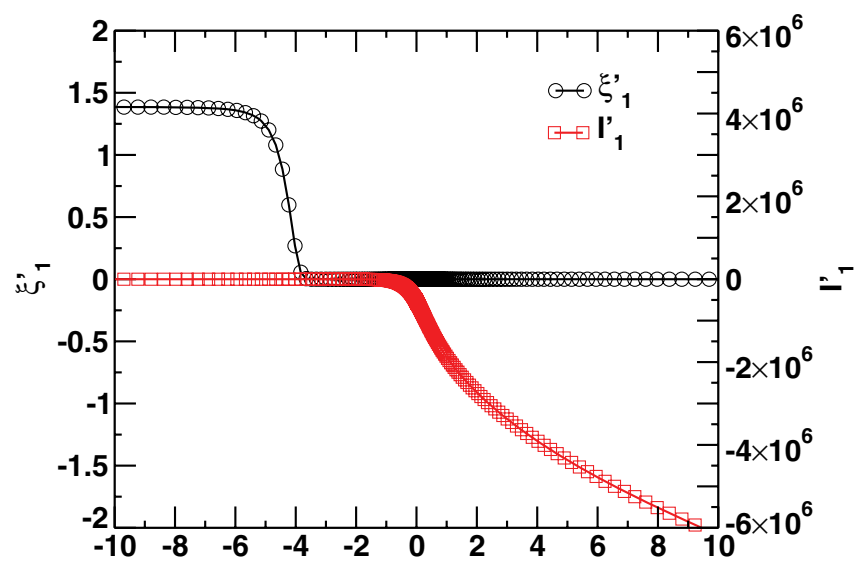

FIG. 15. (Color online) Comparison between $I_{1}^{\prime}$ and $\xi_{1}^{\prime}$ for 500 iterations and $T=10^{-4} T_{K}$. Parameters were chosen to be the same as in Fig. 1. For very low temperatures $\xi_{1}^{\prime}$ (circles, left $y$ axis) exhibits an exponential drop beyond a certain rapidity $\Lambda_{0}(\approx-4$ for the case shown), which is difficult to capture with a fixed grid. This problem can be overcome by using the smooth function $I_{1}^{\prime}$ (squares, right $y$ axis) to calculate $\xi_{1}^{\prime}$ via Eq. (B4c). 
${ }^{1}$ A. C. Hewson, The Kondo Problem to Heavy Fermions (Cambridge University Press, Cambridge, 1997).

${ }^{2}$ A. Zawadowski, Phys. Rev. Lett. 45, 211 (1980).

${ }^{3}$ A. Caldeira and A. Leggett, Ann. Phys. 149, 374 (1983).

${ }^{4}$ A. J. Leggett, S. Chakravarty, A. T. Dorsey, M. P. A. Fisher,

A. Garg, and W. Zwerger, Rev. Mod. Phys. 59, 1 (1987).

${ }^{5}$ U. Weiss, Quantum Dissipative Systems, Vol. 13 (World Scientific, Singapore, 2008).

${ }^{6}$ G. Zaránd, Phys. Rev. B 72, 245103 (2005).

${ }^{7}$ D. Loss and D. P. DiVincenzo, Phys. Rev. A 57, 120 (1998).

${ }^{8}$ J. Park, A. Pasupathy, J. Goldsmith, C. Chang, Y. Yaish, J. Petta, M. Rinkoski, J. Sethna, H. Abruna, P. McEuen, and D. Ralph, Nature (London) 417, 722 (2002).

${ }^{9}$ L. H. Yu and D. Natelson, Nano Lett. 4, 79 (2004).

${ }^{10}$ N. Roch, S. Florens, T. A. Costi, W. Wernsdorfer, and F. Balestro, Phys. Rev. Lett. 103, 197202 (2009).

${ }^{11}$ J. J. Parks, A. R. Champagne, T. A. Costi, W. W. Shum, A. N. Pasupathy, E. Neuscamman, S. Flores-Torres, P. S. Cornaglia, A. A. Aligia, C. A. Balseiro, G. K.-L. Chan, H. D. Abruña, and D. C. Ralph, Science 328, 1370 (2010).

${ }^{12}$ D. Goldhaber-Gordon, J. Göres, M. A. Kastner, H. Shtrikman, D. Mahalu, and U. Meirav, Phys. Rev. Lett. 81, 5225 (1998).

${ }^{13}$ S. M. Cronenwett, T. H. Oosterkamp, and L. P. Kouwenhoven, Science 281, 540 (1998).

${ }^{14}$ A. V. Kretinin, H. Shtrikman, D. Goldhaber-Gordon, M. Hanl, A. Weichselbaum, J. von Delft, T. Costi, and D. Mahalu, Phys. Rev. B 84, 245316 (2011).

${ }^{15}$ J. Nygard, D. Cobden, and P. Lindelof, Nature (London) 408, 342 (2000).

${ }^{16}$ V. Madhavan, W. Chen, T. Jamneala, M. Crommie, and N. Wingreen, Science 280, 567 (1998).

${ }^{17}$ A. Otte, M. Ternes, K. Von Bergmann, S. Loth, H. Brune, C. Lutz, C. Hirjibehedin, and A. Heinrich, Nat. Phys. 4, 847 (2008).

${ }^{18}$ J. Li, W.-D. Schneider, R. Berndt, and B. Delley, Phys. Rev. Lett. 80, 2893 (1998).

${ }^{19}$ W. Metzner and D. Vollhardt, Phys. Rev. Lett. 62, 324 (1989).

${ }^{20}$ A. Georges, G. Kotliar, W. Krauth, and M. J. Rozenberg, Rev. Mod. Phys. 68, 13 (1996).

${ }^{21}$ G. Kotliar and D. Vollhardt, Phys. Today 57, 53 (2004).

${ }^{22}$ D. Vollhardt, Ann. Phys. 524, 1 (2012).

${ }^{23}$ K. G. Wilson, Rev. Mod. Phys. 47, 773 (1975).

${ }^{24}$ H. R. Krishnamurthy, J. W. Wilkins, and K. G. Wilson, Phys. Rev. B 21, 1003 (1980).

${ }^{25}$ H. R. Krishnamurthy, J. W. Wilkins, and K. G. Wilson, Phys. Rev. B 21, 1044 (1980).

${ }^{26}$ R. Bulla, T. A. Costi, and T. Pruschke, Rev. Mod. Phys. 80, 395 (2008).

${ }^{27}$ L. N. Oliveira and J. W. Wilkins, Phys. Rev. Lett. 47, 1553 (1981).

${ }^{28}$ H. O. Frota and L. N. Oliveira, Phys. Rev. B 33, 7871 (1986).

${ }^{29}$ O. Sakai, Y. Shimizu, and T. Kasuya, J. Phys. Soc. Jpn. 58, 3666 (1989).

${ }^{30}$ T. A. Costi and A. C. Hewson, Philos. Mag. B 65, 1165 (1992).

${ }^{31}$ R. Bulla, A. C. Hewson, and T. Pruschke, J. Phys.: Condens. Matter 10, 8365 (1998).

${ }^{32}$ W. Hofstetter, Phys. Rev. Lett. 85, 1508 (2000).

${ }^{33}$ F. B. Anders and A. Schiller, Phys. Rev. Lett. 95, 196801 (2005).

${ }^{34}$ R. Peters, T. Pruschke, and F. B. Anders, Phys. Rev. B 74, 245114 (2006).

${ }^{35}$ A. Weichselbaum and J. von Delft, Phys. Rev. Lett. 99, 076402 (2007).
${ }^{36}$ T. A. Costi, A. C. Hewson, and V. Zlatić, J. Phys.: Condens. Matter 6, 2519 (1994).

${ }^{37}$ C. Gonzalez-Buxton and K. Ingersent, Phys. Rev. B 57, 14254 (1998).

${ }^{38}$ H. v. Löhneysen, A. Rosch, M. Vojta, and P. Wölfle, Rev. Mod. Phys. 79, 1015 (2007).

${ }^{39}$ S. C. Costa, C. A. Paula, V. L. Líbero, and L. N. Oliveira, Phys. Rev. B 55, 30 (1997).

${ }^{40}$ A. M. Tsvelick and P. B. Wiegmann, Phys. Lett. A 89, 368 (1982).

${ }^{41}$ P. B. Wiegmann and A. M. Tsvelick, J. Phys. C 16, 2281 (1983).

${ }^{42}$ A. Okiji and N. Kawakami, Phys. Rev. Lett. 50, 1157 (1983).

${ }^{43}$ Y. Nagaoka, Phys. Rev. 138, A1112 (1965).

${ }^{44}$ P. E. Bloomfield and D. R. Hamann, Phys. Rev. 164, 856 (1967).

${ }^{45}$ B. Kjöllerström, D. J. Scalapino, and J. R. Schrieffer, Phys. Rev. 148, 665 (1966).

${ }^{46}$ V. T. Rajan, J. H. Lowenstein, and N. Andrei, Phys. Rev. Lett. 49, 497 (1982).

${ }^{47}$ H.-U. Desgranges, J. Phys. C 18, 5481 (1985).

${ }^{48}$ P. D. Sacramento and P. Schlottmann, Phys. Rev. B 43, 13294 (1991).

${ }^{49}$ C. J. Bolech and N. Andrei, Phys. Rev. B 71, 205104 (2005).

${ }^{50}$ W. C. Oliveira and L. N. Oliveira, Phys. Rev. B 49, 11986 (1994).

${ }^{51}$ V. L. Campo and L. N. Oliveira, Phys. Rev. B 72, 104432 (2005).

${ }^{52}$ J. B. Silva, W. L. C. Lima, W. C. Oliveira, J. L. N. Mello, L. N. Oliveira, and J. W. Wilkins, Phys. Rev. Lett. 76, 275 (1996).

${ }^{53}$ V. L. Campo and L. N. Oliveira, Phys. Rev. B 70, 153401 (2004).

${ }^{54}$ J. V. B. Ferreira, A. I. I. Ferreira, A. H. Leite, and V. L. Líbero, J. Magn. Magn. Mater. 324, 1011 (2012).

${ }^{55}$ P. W. Anderson, Phys. Rev. 124, 41 (1961).

${ }^{56}$ J. R. Schrieffer and P. A. Wolff, Phys. Rev. 149, 491 (1966).

${ }^{57}$ G. Iche and A. Zawadowski, Solid State Commun. 10, 1001 (1972).

${ }^{58}$ A. C. Hewson, J. Bauer, and W. Koller, Phys. Rev. B 73, 045117 (2006).

${ }^{59}$ L. R. Ramos, W. C. Oliveira, and V. L. Líbero, Phys. Rev. B 67, 085104 (2003)

${ }^{60}$ F. Guinea, V. Hakim, and A. Muramatsu, Phys. Rev. B 32, 4410 (1985).

${ }^{61}$ T. A. Costi and C. Kieffer, Phys. Rev. Lett. 76, 1683 (1996).

${ }^{62}$ T. A. Costi and G. Zarand, Phys. Rev. B 59, 12398 (1999).

${ }^{63}$ T. A. Costi, Phys. Rev. Lett. 80, 1038 (1998).

${ }^{64}$ R. Görlich and U. Weiss, Phys. Rev. B 38, 5245 (1988).

${ }^{65}$ N. Chandra, M. Kollar, and D. Vollhardt, Phys. Rev. B 59, 10541 (1999).

${ }^{66}$ T. A. Costi and R. H. McKenzie, Phys. Rev. A 68, 034301 (2003).

${ }^{67}$ K. L. Hur, Ann. Phys. 323, 2208 (2008).

${ }^{68}$ S. Florens, A. Freyn, D. Venturelli, and R. Narayanan, Phys. Rev. B 84, 155110 (2011).

${ }^{69}$ P. Hänggi and G.-L. Ingold, Acta Phys. Pol. B 37, 1537 (2006).

${ }^{70}$ P. Hänggi, G.-L. Ingold, and P. Talkner, New J. Phys. 10, 115008 (2008).

${ }^{71}$ G.-L. Ingold, P. Hänggi, and P. Talkner, Phys. Rev. E 79, 061105 (2009).

${ }^{72}$ G. Ingold, Eur. Phys. J. B 85, 1 (2012).

${ }^{73}$ G. W. Ford and R. F. O'Connell, Physica E 29, 82 (2005).

${ }^{74}$ H. Hasegawa, J. Math. Phys. 52, 123301 (2011).

${ }^{75} \mathrm{H}$. B. Callen, Thermodynamics and an Introduction to Thermostatistics (John Wiley, New York, 1985).

${ }^{76}$ S. Florens and A. Rosch, Phys. Rev. Lett. 92, 216601 (2004).

${ }^{77}$ R. Žitko and T. Pruschke, Phys. Rev. B 79, 012507 (2009).

${ }^{78}$ M. Vojta and R. Bulla, Eur. Phys. J. B 28, 283 (2002). 
${ }^{79}$ E. Gull, A. J. Millis, A. I. Lichtenstein, A. N. Rubtsov, M. Troyer, and P. Werner, Rev. Mod. Phys. 83, 349 (2011).

${ }^{80}$ J. E. Hirsch and R. M. Fye, Phys. Rev. Lett. 56, 2521 (1986).

${ }^{81}$ A. Liebsch and H. Ishida, J. Phys.: Condens. Matter 24, 053201 (2012).

${ }^{82}$ E. Jakobi, N. Blümer, and P. van Dongen, Phys. Rev. B 80, 115109 (2009).

${ }^{83}$ V. Janiš, Z. Phys. B 83, 227 (1991).

${ }^{84}$ A. Kauch and K. Byczuk, Physica B 407, 209 (2012).

${ }^{85}$ D. E. Logan, M. P. Eastwood, and M. A. Tusch, J. Phys.: Condens. Matter 10, 2673 (1998).

${ }^{86}$ A. J. Daley, C. Kollath, U. Schollwöck, and G. Vidal, J. Stat. Mech. (2004) P04005.
${ }^{87}$ S. R. White and A. E. Feiguin, Phys. Rev. Lett. 93, 076401 (2004).

${ }^{88}$ L. G. G. V. Dias da Silva, F. Heidrich-Meisner, A. E. Feiguin, C. A. Büsser, G. B. Martins, E. V. Anda, and E. Dagotto, Phys. Rev. B 78, 195317 (2008).

${ }^{89}$ T. A. Costi, Phys. Rev. B 55, 3003 (1997).

${ }^{90}$ A. Rosch, Eur. Phys. J. B 85, 1 (2012).

${ }^{91}$ N. Kawakami and A. Okiji, Phys. Lett. A 86, 483 (1981).

${ }^{92}$ N. Kawakami and A. Okiji, J. Phys. Soc. Jpn. 51, 2043 (1982).

${ }^{93}$ A. M. Tsvelick and P. B. Wiegmann, J. Phys. C 16, 2321 (1983).

${ }^{94}$ V. M. Filyov, A. M. Tsvelick, and P. B. Wiegmann, Phys. Lett. A 89, 157 (1982).

${ }^{95}$ M. Takahashi and M. Shiroishi, Phys. Rev. B 65, 165104 (2002).

${ }^{96}$ N. Kawakami and A. Okiji, Solid State Commun. 43, 467 (1982). 